\title{
$9-2016$
}

\section{Progestogen-only contraceptive use among breastfeeding women: a systematic review.}

\author{
Sharon J. Phillips \\ World Health Organization \\ Naomi K. Tepper \\ US Centers for Disease Control and Prevention \\ Nathalie Kapp \\ Reproductive Health Consultant \\ Kavita Nanda \\ FHI 360 \\ Marleen Temmerman \\ Aga Khan University, marleen.temmerman@aku.edu
}

See next page for additional authors

Follow this and additional works at: https://ecommons.aku.edu/eastafrica_fhs_mc_obstet_gynaecol

Part of the Obstetrics and Gynecology Commons

\section{Recommended Citation}

Phillips, S. J., Tepper, N. K., Kapp, N., Nanda, K., Temmerman, M., Curtis, K. M. (2016). Progestogen-only contraceptive use among breastfeeding women: a systematic review.. Contraception, 94(3), 226-252. Available at: https://ecommons.aku.edu/eastafrica_fhs_mc_obstet_gynaecol/193 


\section{Authors}

Sharon J. Phillips, Naomi K. Tepper, Nathalie Kapp, Kavita Nanda, Marleen Temmerman, and Kathryn M. Curtis 


\title{
Progestogen-only contraceptive use among breastfeeding women: a systematic review
}

\author{
Sharon J. Phillips ${ }^{\mathrm{a}, *}$, Naomi K. Tepper ${ }^{\mathrm{b}}$, Nathalie Kapp ${ }^{\mathrm{c}}$, Kavita Nanda ${ }^{\mathrm{d}}$, \\ Marleen Temmerman ${ }^{\mathrm{a}}$, Kathryn M. Curtis ${ }^{\mathrm{b}}$ \\ ${ }^{a}$ Department of Reproductive Health and Research, World Health Organization, Geneva, Switzerland \\ ${ }^{\mathrm{b}}$ Division of Reproductive Health, US Centers for Disease Control and Prevention, Atlanta, GA, USA \\ ${ }^{\mathrm{c}}$ Reproductive Health Consultant, Paris, France \\ ${ }^{\mathrm{d}}$ FHI 360, Durham, NC, USA
}

Received 7 May 2015; revised 20 September 2015; accepted 21 September 2015

\begin{abstract}
Background: Postpartum women need effective contraception. Concerns have been raised that use of progestogen-only contraceptives (POCs) may affect breastfeeding performance and infant health outcomes.

Objectives: We investigated the clinical outcomes of breastfeeding duration, initiation of supplemental feeding and weaning, as well as infant outcomes including infant growth, health and development among breastfeeding women using POCs compared with breastfeeding women not using POCs.

Search strategy: We searched the PubMed database for all articles published from database inception through December 2014.

Selection criteria: We included primary research studies of breastfeeding women of any age or parity who received POCs, including progestogen-only pills, injectables, implants or hormonal intrauterine devices (IUDs). The main outcomes were breastfeeding performance (as measured by initiation, continuation, frequency and exclusivity of breastfeeding) and infant health (as measured by growth, development or adverse health effects).

Results: Forty-nine articles reporting on 47 different studies were identified that investigated the use of POCs in breastfeeding women and reported clinically relevant outcomes of infant growth, health or breastfeeding performance. Studies ranged from poor to fair methodological quality and generally failed to show negative effects of the use of POCs on breastfeeding outcomes or on infant growth or development. One randomized controlled trial (RCT) raises concerns that immediate insertion of the levonorgestrel IUD postpartum may be associated with poorer breastfeeding performance when compared with delayed insertion, although two other RCTs evaluating early etonogestrel implants compared with delayed initiation of implants or depot medroxyprogesterone acetate failed to find such an association.

Conclusion: The preponderance of evidence fails to demonstrate adverse breastfeeding outcomes or negative health outcomes in infants such as restricted growth, health problems or impaired development. Evidence newly added to this review was largely consistent with previous evidence. (C) 2016 Published by Elsevier Inc. This is an open access article under the CC BY-NC-ND license (http://creativecommons.org/licenses/by-nc-nd/4.0/).
\end{abstract}

Keywords: Lactation; Contraception; Progestogens; Breastfeeding

\section{Introduction}

The benefits of breastfeeding for both women and their infants are considerable [1-3]. The World Health Organization

\footnotetext{
The findings and conclusions in this report are those of the authors and do not necessarily represent the official position of the World Health Organization or US Centers for Disease Control and Prevention.

* Corresponding author.

E-mail addresses: sjp633@mail.harvard.edu, sharonphillipsmd@gmail.com (S.J. Phillips).
}

(WHO) recommends infants breastfeed exclusively during the first months of life [4]. Although women breastfeeding exclusively and on demand are unlikely to conceive before 6 weeks postpartum, many women discontinue fully breastfeeding before that time and are at risk of repeat pregnancy [5]. Because birth spacing has demonstrated health benefits for women and infants, early initiation of contraception in the postpartum period may improve outcomes.

Progestogen-only and progesterone contraceptives have been in use for years; however, their dosages and formulations have changed over time. Methods available 
Table 1

Included studies

\begin{tabular}{|c|c|c|c|c|c|c|}
\hline $\begin{array}{l}\text { Author, } \\
\text { year, } \\
\text { source of support }\end{array}$ & $\begin{array}{l}\text { Study design, } \\
\text { location, } \\
\text { population }\end{array}$ & Interventions & $\begin{array}{l}\text { Outcomes, } \\
\text { follow-up duration }\end{array}$ & Results & Strengths/weaknesses & $\begin{array}{l}\text { Quality grading/ } \\
\text { key question }\end{array}$ \\
\hline $\begin{array}{l}\text { Kamal, } 1969[30] \\
\text { Not stated } \\
\text { Newly identified }\end{array}$ & $\begin{array}{l}\text { Nonrandomized clinical } \\
\text { trial } \\
\text { Egypt } \\
N=120 \text { PP women } \\
\text { (data available on 50) }\end{array}$ & $\begin{array}{l}\text { 6-10 weeks PP: } \\
\text { POP ( } 0.5 \text { mg lynestrenol) } \\
\text { IUD+placebo } \\
2 \text { kinds of COCs, } 1 \text { combined } \\
\text { injectable contraceptive (not } \\
\text { reported here) } \\
\text { Allocation not reported }\end{array}$ & $\begin{array}{l}\text { BF performance (age of } \\
\text { supplementation) } \\
\text { Infant growth (growth } \\
\text { curve, percent weight } \\
\text { increase) } \\
\text { Follow-up } 32 \text { weeks }\end{array}$ & $\begin{array}{l}\frac{\text { BF outcomes }}{\text {-Average age of }} \\
\text { supplementation } \\
11.2 \text { weeks POP } \\
\text { group, } 15 \text { weeks } \\
\text { placebo (statistics not } \\
\text { reported) } \\
\text { Infant outcomes } \\
\text {-No relation between } \\
\text { growth curve and } \\
\text { method used }\end{array}$ & $\begin{array}{l}\text { Strengths } \\
\text {-Double blinded } \\
\text { Weaknesses } \\
\text {-No statistical analysis } \\
\text { reported for comparisons } \\
\text { of interest } \\
\text {-High, but not clearly } \\
\text { reported, loss to } \\
\text { follow-up } \\
\text {-Number of participants/ } \\
\text { group not reported }\end{array}$ & $\begin{array}{l}\text { Level II-1 } \\
\text { Poor } \\
\text { Key Question } 1\end{array}$ \\
\hline $\begin{array}{l}\text { Kamal, } 1970[43] \\
\text { Not stated }\end{array}$ & $\begin{array}{l}\text { Nonrandomized } \\
\text { clinical trial } \\
\text { Egypt } \\
N=40 \text { primiparous and } \\
\text { multiparous women, } \\
\text { ages } 20-37 \text { years }\end{array}$ & $\begin{array}{l}2 \text { days PP: } \\
10=\text { placebo } \\
10=\text { POP (lynestrenol } \\
500 \mathrm{mcg} \text { ) } \\
10=\mathrm{COC} \text { (results not } \\
\text { presented) } \\
10=\text { ethinyl estradiol (results } \\
\text { not presented) }\end{array}$ & $\begin{array}{l}\text { BF performance (initiation } \\
\text { of lactation) } \\
\text { Infant growth (weight) } \\
\text { Follow-up } 14 \text { days }\end{array}$ & $\begin{array}{l}\text { BF outcomes } \\
\text {-Lactation initiation } \\
\text { earlier ( } 3 \text { vs. } 5 \text { days) } \\
\text { in POP than placebo } \\
\text { group } \\
\text { Infant outcomes } \\
\text {-Greatest weight } \\
\text { increase in } \\
\text { POP-exposed infants }\end{array}$ & $\begin{array}{l}\text { Strengths: } \\
\text {-Included primiparas } \\
\text { Weaknesses: } \\
\text {-Nonrandomized } \\
\text {-Small sample size } \\
\text {-Short follow-up } \\
\text {-No statistical } \\
\text { comparisons }\end{array}$ & $\begin{array}{l}\text { Level II-1 } \\
\text { Poor } \\
\text { Key Question } 1\end{array}$ \\
\hline $\begin{array}{l}\text { Karim, } 1971[36] \\
\text { Not stated }\end{array}$ & $\begin{array}{l}\text { Prospective cohort } \\
\text { Egypt } \\
N=331 \text { women after } \\
\text { normal delivery }\end{array}$ & $\begin{array}{l}7 \text { days PP: } \\
68=\text { NET-EN }(200 \mathrm{mg}) \\
51=\text { DMPA }(150 \mathrm{mg}) \\
100=\mathrm{NH} \\
42 \text { days PP: } \\
57=\text { NET-EN } \\
55=\text { DMPA }\end{array}$ & $\begin{array}{l}\text { BF performance } \\
\text { (supplementation) } \\
\text { Infant growth and health } \\
\text { (weight, physical exam, } \\
\text { dentition, mentality, } \\
\text { walking, radiographs) } \\
\text { Follow-up } 18 \text { months }\end{array}$ & $\begin{array}{l}\frac{\text { BF outcomes }}{- \text { No BF }} \\
\text { supplementation } \\
\text { reported up to } \\
6 \text { months in any } \\
\text { groups } \\
\text { Infant outcomes } \\
\text {-After 3rd month, } \\
\text { infant weight gain per } \\
\text { month higher in all } \\
\text { POC groups than in } \\
\text { NH controls; weight } \\
\text { gain in hormonal } \\
\text { groups equivalent } \\
\text {-No physical, mental } \\
\text { or radiologic } \\
\text { differences in infants } \\
\text { between groups }\end{array}$ & $\begin{array}{l}\text { Weaknesses: } \\
\text {-Percent follow- up of } \\
\text { infants not reported } \\
\text {-No standardized } \\
\text { techniques to measure } \\
\text { health and specifics of } \\
\text { health outcomes not } \\
\text { reported }\end{array}$ & $\begin{array}{l}\text { Level II-2, } \\
\text { poor } \\
\text { Key Questions } 1 \text { and } 2\end{array}$ \\
\hline $\begin{array}{c}\text { Guiloffetal., } 1974 \text { [37] } \\
\text { Population council, } \\
\text { Warner-Lambert } \\
\text { Research Institute }\end{array}$ & $\begin{array}{l}\text { Cohort } \\
\text { Chile } \\
N=696 \text { multiparous } \\
\text { women, ages } 16- \\
40 \text { years }\end{array}$ & $\begin{array}{l}\text { 1-2 days PP: } \\
80=\text { DMPA } \\
\text { ( } 250 \mathrm{mg} \text { im q } 6 \text { months }) \\
30 \text { days PP: } \\
\text { 33=DMPA }\end{array}$ & $\begin{array}{l}\text { BF performance (mean } \\
\text { duration of lactation) } \\
\text { Follow-up } 12 \text { months }\end{array}$ & $\begin{array}{l}\text { BF outcomes } \\
\text { Mean lactation } \\
\text { duration (presented } \\
\text { as mean months with } \\
95 \% \text { CI) }\end{array}$ & $\begin{array}{l}\text { Weaknesses: } \\
\text {-Unclear if prospective } \\
\text { or retrospective } \\
\text {-Historical control } \\
\text {-Historical recollection }\end{array}$ & $\begin{array}{l}\text { Level II-2 } \\
\text { Poor } \\
\text { Key Question } 1\end{array}$ \\
\hline
\end{tabular}




Study design,

\begin{tabular}{|c|c|c|c|c|c|c|}
\hline $\begin{array}{l}\text { Author, } \\
\text { year, } \\
\text { source of support }\end{array}$ & $\begin{array}{l}\text { Study design, } \\
\text { location, } \\
\text { population }\end{array}$ & Interventions & $\begin{array}{l}\text { Outcomes, } \\
\text { follow-up duration }\end{array}$ & Results & Strengths/weaknesses & $\begin{array}{l}\text { Quality grading/ } \\
\text { key question }\end{array}$ \\
\hline & $\begin{array}{l}\text { Historical control was } \\
\text { composed of the past } \\
\text { lactation history of a } \\
\text { subset of women } \\
\text { enrolled in the study } \\
\text { who were still BF at } \\
30 \text { days }\end{array}$ & $\begin{array}{l}54=\text { Chlormadione acetate } \\
\text { ( } 250 \mathrm{mg} \text { im q } 3 \text { months) } \\
81=\text { Quingestanol acetate } \\
(300 \mathrm{mcg}) \\
81=\text { IUD } \\
\text { Other participants used } \\
\text { COCs (results not reported } \\
\text { here) }\end{array}$ & & $\begin{array}{l}\text { DMPA } 1-2 \text { days PP: } \\
6.7(5.2-8.7) \\
\text { Historical control: } 4.8 \\
\text { (4.1-5.3) } \\
\text { DMPA } 30 \text { days PP: } \\
\text { 9.3 (6.0-10.0) } \\
\text { Chlormadione acetate } \\
\text { 30 days PP: } \\
7.5 \text { months (4.7-9.7) } \\
\text { Quingestanol acetate } \\
\text { 30 days PP: } 4.2 \\
\text { (2.8-5.6) } \\
\text { IUD 30 days PP: } 7.7 \\
\text { (6.8-8.9) } \\
\text { Historical control: } 5.3 \\
\text { (4.8-5.8) }\end{array}$ & of duration of lactation & \\
\hline $\begin{array}{l}\text { Giner-Velasquez } \\
\text { et al., } 1976 \text { [33] } \\
\text { Not stated }\end{array}$ & $\begin{array}{l}\text { RCT } \\
\text { Mexico } \\
N=20 \text { healthy } \\
\text { women, ages } \\
18-36 \text { years }\end{array}$ & $\begin{array}{l}\text { <14 h PP: } \\
\text { 12=NET }(350 \mathrm{mcg}) \\
\text { 8=Placebo }\end{array}$ & $\begin{array}{l}\text { BF performance (initiation) } \\
\text { Infant growth (weight) } \\
\text { Follow-up } 14 \text { days }\end{array}$ & $\begin{array}{l}\text { BF outcomes } \\
\text { No difference } \\
\text { between groups in BF } \\
\text { initiation (statistics } \\
\text { not reported) } \\
\text { Infant outcomes } \\
\text { No difference } \\
\text { between groups in } \\
\text { weight gain (average } \\
493 \mathrm{~g} \text { placebo, } 441 \mathrm{~g} \\
\text { NET, difference not } \\
\text { significant) }\end{array}$ & $\begin{array}{l}\text { Weaknesses: } \\
\text {-Methods poorly described } \\
\text {-Small sample size } \\
\text {-Follow-up and } \\
\text { exclusions not described }\end{array}$ & $\begin{array}{l}\text { Level I, } \\
\text { Poor } \\
\text { Key Question } 1\end{array}$ \\
\hline $\begin{array}{l}\text { Zanartu et al., } 1976 \text { [31] } \\
\text { CEBRE, University } \\
\text { of Chile Medical } \\
\text { School } \\
\text { Newly identified }\end{array}$ & $\begin{array}{l}\text { Prospective cohort } \\
N=406 \text { fully BF } \\
\text { women using DMPA } \\
\text { with at least } \\
18 \text { months follow-up, } \\
173 \text { controls }\end{array}$ & $\begin{array}{l}\text { First } 30 \text { days PP: } \\
N=133 \text { DMPA } \\
30-90 \text { days PP: } \\
N=206 \text { DMPA } 30-90 \text { days } \\
\text { PP } \\
91-180 \text { days PP: } \\
N=67 \text { DMPA } \\
\text { (DMPA } 150 \text { or } 250-300 \mathrm{mg} \text { ) } \\
N=173 \text { no DMPA (and either } \\
\text { received education about BF } \\
\text { or no intervention) }\end{array}$ & $\begin{array}{l}\text { BF performance (exclusive } \\
\text { and partial lactation status at } \\
3,6,12,18 \text { months) } \\
\text { Follow-up } 18 \text { months }\end{array}$ & $\begin{array}{l}\text { BF outcomes } \\
\text { 3rd month/6th month } \\
\text { PP: } 94 \% / 80 \% \text { DMPA } \\
\text { group fully BF; fewer } \\
\text { in non-DMPA group } \\
\text { (p<.001) } \\
12 \text { th } / 18 \text { th month PP: } \\
42 \% / 10 \% \text { still BF; } \\
\text { fewer in non-DMPA } \\
\text { group (p }<.001) \\
\text { Of those who received } \\
\text { DMPA up to } 90 \text { days } \\
\text { PP, } 35 \% \text { still BF at } \\
12 \text { months (vs. } 64 \% \\
\text { who received after } \\
90 \text { days, no statistics) }\end{array}$ & $\begin{array}{l}\text { Strengths: } \\
\text {-High percentage with } \\
\text { follow-up ( } 406 / 500 \text { with } \\
\text { at least } 18 \text { months } \\
\text { follow-up) } \\
\text { Weaknesses: } \\
\text {-Unclear if non-DMPA } \\
\text { users were using other } \\
\text { hormonal or NH } \\
\text { contraceptives } \\
\text {-No separate analysis by } \\
\text { DMPA dose; minimal } \\
\text { analysis by timing; no } \\
\text { statistical analysis for } \\
\text { indirect comparison } \\
\text {-Wide range in timing of } \\
\text { DMPA administration }\end{array}$ & $\begin{array}{l}\text { Level II-2, Poor } \\
\text { Key Question } 1\end{array}$ \\
\hline
\end{tabular}

Outcomes,




\begin{tabular}{|c|c|c|c|c|c|c|}
\hline $\begin{array}{l}\text { Zanartu et al., } 1976 \\
\text { [45] } \\
\text { Ayerst }\end{array}$ & $\begin{array}{l}\text { Nonrandomized } \\
\text { clinical trial } \\
\text { Chile } \\
N=100 \text { healthy } \\
\text { women, ages } 19-42 \text { years }\end{array}$ & $\begin{array}{l}\text { 3rd to 10th week PP: } \\
100=\text { Chlormadione acetate } \\
0.6 \mathrm{mg} \\
173=\mathrm{NH} \text { (historical control; some } \\
\text { inert IUD, some no method) }\end{array}$ & $\begin{array}{l}\text { BF performance (duration) } \\
\text { Follow-up } 18 \text { months }\end{array}$ & $\begin{array}{l}\text { BF outcomes } \\
\text { At } 3 \text { months: } \\
98 \% \text { Chlormadione } \\
\text { still BF } \\
76 \% \mathrm{NH} \text { still BF } \\
\text { At } 6 \text { months: } \\
80 \% \text { (POP) and } 56 \% \\
(\mathrm{NH}) \text { still BF } \\
\text { At } 12 \text { months: } \\
42 \%(\mathrm{POP}) \text { and } 0 \% \\
(\mathrm{NH}) \text { still } \mathrm{BF} \\
(\mathrm{p}<.001)\end{array}$ & $\begin{array}{l}\text {-No statistical analyses } \\
\text { Weaknesses: } \\
\text {-Historical control } \\
\text {-Wide variation in } \\
\text { timing of contraceptive } \\
\text { initiation } \\
\text {-Loss to follow-up not } \\
\text { reported } \\
\text {-Statistical analyses not } \\
\text { reported for all outcomes } \\
\text { of interest }\end{array}$ & $\begin{array}{l}\text { Level II-1, } \\
\text { Poor } \\
\text { Key Question } 1\end{array}$ \\
\hline $\begin{array}{l}\text { Seth et al., } 1977 \text { [49] } \\
\text { WHO }\end{array}$ & $\begin{array}{l}\text { Cohort } \\
\text { India } \\
N=50 \text { healthy, } \\
\text { women, ages } \\
20-40 \text { years }\end{array}$ & $\begin{array}{l}6 \text { days PP: } \\
23=\text { Implant ( } 40 \mathrm{mg} \\
\text { norethindrone acetate) } \\
\text { (early) } \\
6 \text { weeks PP: } \\
12=\text { Implant (delayed) } \\
15=\text { NH (Condoms/gel) }\end{array}$ & $\begin{array}{l}\text { BF performance (continued } \\
\text { BF at } 8 \text { months, } \\
\text { supplementation rates) } \\
\text { Infant growth (weight) } \\
\text { Follow-up } 11 \text { months }\end{array}$ & $\begin{array}{l}\frac{\text { BF outcomes }}{\text { Still BF at } 8 \text { months }} \\
80 \% \mathrm{NH}, 56.6 \% \\
\text { early, } 66.6 \% \text { delayed, } \\
\text { difference not } \\
\text { significant } \\
3 \text { months } \\
\text { supplementation } \\
\text { Early implant } 56.4 \% \text {, } \\
\text { controls } 40 \% \text { (p<.05), } \\
\text { other times, NS } \\
\text { Infant outcomes } \\
\text { No differences between } \\
\text { groups in weight }\end{array}$ & $\begin{array}{l}\text { Weaknesses: } \\
\text {-Small sample size } \\
\text {-Methods poorly } \\
\text { described } \\
\text {-Baseline characteristics } \\
\text { not described } \\
\text {-Percent follow-up not } \\
\text { reported }\end{array}$ & $\begin{array}{l}\text { Level II-2, Poor } \\
\text { Key Questions } 1 \text { and } 2\end{array}$ \\
\hline $\begin{array}{l}\text { Croxatto et al., } 1982 \\
\text { [55] } \\
\text { Population Council } \\
\text { and Canadian } \\
\text { International } \\
\text { Development } \\
\text { Research Center }\end{array}$ & $\begin{array}{l}\text { Cohort } \\
\text { Chile } \\
N=439 \text { healthy } \\
\text { women who did not } \\
\text { hold jobs, ages } \\
18-35 \text { years }\end{array}$ & $\begin{array}{l}30-35 \text { days PP: } \\
84=\text { Progesterone pellets } \\
(100 \mathrm{mg}) \\
130=\text { Placebo injectable } \\
125=\text { Cu T200 IUD }\end{array}$ & $\begin{array}{l}\text { BF performance (fully, } \\
\text { partially or not BF at } \\
\text { follow-up visits) } \\
\text { Infant growth (weight) } \\
\text { Infant health (reports of } \\
\text { intercurrent illness) } \\
\text { Follow-up } 12 \text { months }\end{array}$ & $\begin{array}{l}\text { BF outcomes } \\
\text { Fully } B F: \text { No } \\
\text { significant difference } \\
\text { between groups at } 3,6 \\
\text { or } 9 \text { months } \\
B F \text { at } 6 \text { months } \\
51.2 \% \text { progesterone } \\
58.3 \% \text { IUD } \\
B F \text { at } 12 \text { months } \\
10.7 \% \text { progesterone } \\
17.6 \% \text { IUD (p }<.05 \text { ) } \\
\text { Infant outcomes } \\
\text { No differences in } \\
\text { infant weight gain } \\
\text { among groups } \\
\text { (4515 g progesterone, } \\
4633 \text { placebo, } 4801 \\
\text { IUD, not statistically } \\
\text { significant) or health } \\
\text { (no statistics reported) }\end{array}$ & $\begin{array}{l}\text { Weaknesses: } \\
\text {-Little description of } \\
\text { intercurrent illnesses (or } \\
\text { their assessment) } \\
\text {-High rates of } \\
\text { discontinuation/ } \\
\text { termination from study/ } \\
\text { loss to follow-up in all } \\
\text { groups }\end{array}$ & $\begin{array}{l}\text { Level II-2, Fair } \\
\text { Key Question } 1\end{array}$ \\
\hline $\begin{array}{l}\text { Dahlberg, } 1982 \text { [32] } \\
\text { No funding }\end{array}$ & $\begin{array}{l}\text { Retrospective cohort } \\
\text { Thailand }\end{array}$ & $\begin{array}{l}\text { Some time in } 1 \text { st } 9 \text { months PP: } \\
210=\text { Some exposure to }\end{array}$ & $\begin{array}{l}\text { Infant growth (weight) } \\
\text { Infant health (incidence of }\end{array}$ & $\frac{\text { Infant outcomes }}{\text { Weight gain }}$ & $\frac{\text { Strengths: }}{\text {-Subgroup data }}$ & $\begin{array}{l}\text { Level II-2, Poor } \\
\text { Key Questions } 1 \text { and } 2\end{array}$ \\
\hline
\end{tabular}




\begin{tabular}{|c|c|c|c|c|c|c|}
\hline $\begin{array}{l}\text { Author, } \\
\text { year, } \\
\text { source of support }\end{array}$ & $\begin{array}{l}\text { Study design, } \\
\text { location, } \\
\text { population }\end{array}$ & Interventions & $\begin{array}{l}\text { Outcomes, } \\
\text { follow-up duration }\end{array}$ & Results & Strengths/weaknesses & $\begin{array}{l}\text { Quality grading/ } \\
\text { key question }\end{array}$ \\
\hline Newly identified & $\begin{array}{l}N=331 \text { infants born at } \\
\text { Thai hospital between } \\
1977 \text { and } 1979\end{array}$ & $\begin{array}{l}\text { DMPA } \\
\text { 121=No exposure to DMPA }\end{array}$ & $\begin{array}{l}\text { infectious diseases leading } \\
\text { to clinic visits) } \\
\text { Follow-up up to } 46 \text { months }\end{array}$ & $\begin{array}{l}\text { No difference } \\
\text { between groups at any } \\
\text { time point in } \\
\text { follow-up, regardless } \\
\text { of length of exposure } \\
\text { Health } \\
\text { No difference in } \\
\text { average numbers of } \\
\text { infectious diseases } \\
\text { reported per year } \\
\text { between groups } \\
\text { (although subgroup } \\
\text { who received DMPA } \\
\text { at } 2 \text { days PP had } 75 \% \\
\text { higher incidence than } \\
\text { other groups, } \\
\text { statistics not reported) }\end{array}$ & $\begin{array}{l}\text { presented with different } \\
\text { amounts of DMPA } \\
\text { exposure } \\
\text { Weaknesses: } \\
\text {-Data obtained solely } \\
\text { through record review } \\
\text {-Statistical analysis not } \\
\text { reported } \\
\text {-Analytical methods not } \\
\text { clearly described } \\
\text {-Timing of exposure to } \\
\text { DMPA not clear } \\
\text {-Wide variation in when } \\
\text { DMPA was given PP }\end{array}$ & \\
\hline $\begin{array}{l}\text { Heikkila and } \\
\text { Luukkainen, } 1982 \\
\text { [34] } \\
\text { Population Council, } \\
\text { US Agency for } \\
\text { International } \\
\text { Development, Ford } \\
\text { Foundation }\end{array}$ & $\begin{array}{l}\text { RCT (with change to } \\
\text { protocol partway } \\
\text { through trial) } \\
\text { Finland } \\
N=110 \text { women }\end{array}$ & $\begin{array}{l}\text { 32-56 days PP: } \\
\text { 30=LNG }(10 \mathrm{mcg} / \text { day IUD) } \\
40=\mathrm{LNG}(30 \mathrm{mcg} / \text { day IUD }) \\
40=\text { Copper IUD }\end{array}$ & $\begin{array}{l}\text { BF performance (duration, } \\
\text { time to supplementation) } \\
\text { Infant growth (height, } \\
\text { weight) } \\
\text { Infant development (time of } \\
\text { walking, tooth eruption) } \\
\text { Infant health (infectious } \\
\text { diseases) } \\
\text { Follow-up } 12 \text { months }\end{array}$ & 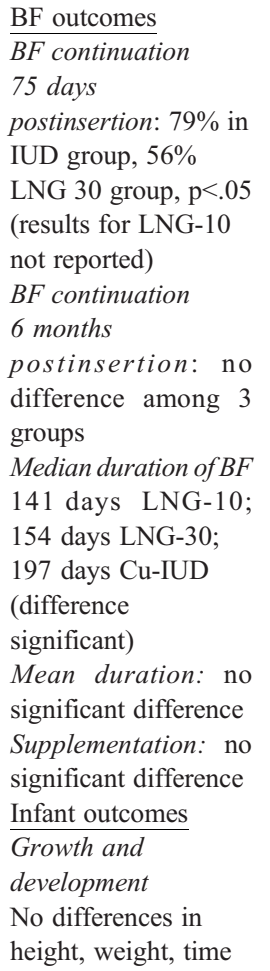 & $\begin{array}{l}\text { Weaknesses: } \\
\text {-Allocation concealment } \\
\text { and randomization } \\
\text { sequence ill-described } \\
\text {-Mid-way through trial, } \\
\text { added lower-dose IUD } \\
\text { and changed allocation } \\
\text { scheme } \\
\text {-Copper IUD group } \\
\text { younger and less parous } \\
\text {-Illnesses not recorded } \\
\text { or assessed systematically }\end{array}$ & $\begin{array}{l}\text { Level I, Poor } \\
\text { Key Question } 1\end{array}$ \\
\hline
\end{tabular}




West et al., 1983 [44]
Medical Research
Council

Diaz et al., 1984 [54]
Instituto Bioquinico
Beta, WHO,
International
Development
Research Centre of
Canada, Population
Council

$\begin{array}{ll}\begin{array}{l}\text { Cohort } \\ \text { Scotland }\end{array} & \begin{array}{l}\text { Up to } 8 \text { weeks PP: } \\ 84=\text { Norethisterone } 0.35 \mathrm{mg} \\ N=227 \text { healthy }\end{array} \\ \begin{array}{l}(76 \% \text { by week } 4) \\ \text { women, fully BF }\end{array} & \begin{array}{l}29=\mathrm{COC} \\ \text { (data available on }\end{array} \\ \begin{array}{l}89=\mathrm{NH} \\ \text { 203) }\end{array} & 30 \text { days PP: } \\ \text { Cohort } & 84=\text { Progesterone pellets } \\ \text { Chile } & (100 \mathrm{mg}) \\ N=653 \text { healthy } & 125=\mathrm{Cu} \text { T200 IUD } \\ \text { women after normal } & 130=\text { Placebo injection } \\ \text { pregnancy, 18-35 years } & 60 \text { days PP: } \\ & 193=\text { Progesterone pellets } \\ & 121=\text { Cu T200 IUD }\end{array}$

\begin{tabular}{|c|c|c|}
\hline $\begin{array}{l}\text { Jimenez et al., } 1984 \\
\text { [38] } \\
\text { Upjohn }\end{array}$ & $\begin{array}{l}\text { Retrospective cohort - } \\
\text { follow-up to } \\
\text { unpublished primary } \\
\text { study } \\
\text { Chile } \\
N=270 \\
\text { Women and their } \\
\text { children exposed to } \\
\text { contraception during } \\
\text { lactation, } \\
\text { 3-6 years prior }\end{array}$ & $\begin{array}{l}\text { 2nd month PP: } \\
128=\text { DMPA } \quad(150 \mathrm{mg} \mathrm{q} \\
3 \text { months }) \\
142=\mathrm{NH}\end{array}$ \\
\hline
\end{tabular}

$121=\mathrm{Cu}$ T200 IUD of walking, tooth

eruption

Health

No differences

between groups in

respiratory/middle

ear infections

BF performance (duration,

BF performance (exclusivity

at 6 months and

continuation)

onths) and health

(how assessed not defined)

Follow-up 6 months

BF performance (reported

duration of lactation)

Infant growth (weight, arm

circumference, head

circumference)

Infant health (respiratory

infections, diarrheal disease,

hospital admissions, mortality)

Infant development

(standardized physical exam,

interview, record review and

psychomotor scale)

60 days PP and their

DMPA vs. 13 months
BF outcomes:

At 3 months: $62 \%$

POP, $62 \% \mathrm{NH}$ still BF

At 5 months: $51 \%$

POP, 53\% NH still BF

(statistics not reported)

BF outcomes:

Weaknesses

-Follow-up by postal

survey

-No statistical analysis

- Unclear when methods

were initiated

Strengths

-Clear description of methods and analysis

Weaknesses:

between those

initiated at 30 or

contemporary

-Women lost to

follow-up or

discontinuing their

method not reported

day 60 were more

likely to supplemen

at month 6 than those

initiating at day 30

$68 \%$ exclusive vs.

$53 \%$ exclusive,

presented for outcomes

of interest

-No control for

confounding

-Unclear how infant

health was assessed

nfant outcomes

Weight gain

No difference

between groups

Health

No negative effects of

progesterone on

infant health

Median lactatio

Median lactar

uration: 21 months

$\mathrm{NH}(\mathrm{p}<.05)$

Infant outcomes

Growth

No difference

between groups in

height; weight

different between

groups but no

Weaknesses:

-Primary study not published

-Some outcomes relied

on retrospective

self-report

-Groups dissimilar

(mothers in DMPA

group older, of higher

parity)
-Statistical analyses no

Level II-2, Poor

Key Question 1

Level II-2, Poor

Key Questions 1 and 2

Level II-2, Poor 


\begin{tabular}{|c|c|c|c|c|c|c|}
\hline $\begin{array}{l}\text { Author, } \\
\text { year, } \\
\text { source of support }\end{array}$ & $\begin{array}{l}\text { Study design, } \\
\text { location, } \\
\text { population }\end{array}$ & Interventions & $\begin{array}{l}\text { Outcomes, } \\
\text { follow-up duration }\end{array}$ & Results & Strengths/weaknesses & $\begin{array}{l}\text { Quality grading/ } \\
\text { key question }\end{array}$ \\
\hline & & & & $\begin{array}{l}\text { difference when } \\
\text { adjusted for } \\
\text { confounders } \\
\text { Health } \\
1 \text { death in control } \\
\text { group (accidental), } 0 \\
\text { in DMPA group } \\
\text { Development } \\
\text { No differences } \\
\text { between groups in } \\
\text { psychomotor } \\
\text { development, } \\
\text { milestones, health } \\
\text { problems, infant } \\
\text { height or physical } \\
\text { exam }\end{array}$ & & \\
\hline $\begin{array}{l}\text { Tankeyoon et al., } \\
1984[57] \\
\text { WHO }\end{array}$ & $\begin{array}{l}\text { Prospective cohort } \\
\text { with nested RCT } \\
\text { Hungary } \\
\text { Thailand } \\
N=341 \text { experienced } \\
\text { BF women, ages } 20- \\
35 \text { years, parity } 2-4 \text {, } \\
\text { after healthy term } \\
\text { delivery }\end{array}$ & $\begin{array}{l}6 \text { weeks PP ( } \pm 3 \text { days): } \\
59=\text { DMPA } \\
111=\mathrm{N} \mathrm{H} \text { (b a r r i e r s, } \\
\text { sterilization, IUD) } \\
\text { Pill-users (randomized): } \\
85=\text { POP } \\
86=\text { COC (results not } \\
\text { reported here) }\end{array}$ & $\begin{array}{l}\text { BF performance (use of } \\
\text { complementary food, } \\
\text { discontinuation due to } \\
\text { perceived inadequate milk } \\
\text { supply) } \\
\text { Infant growth (weight, } \\
\text { length, arm circumference) } \\
\text { Follow-up } 24 \text { weeks }\end{array}$ & $\begin{array}{l}\text { BF outcomes: } \\
\text { No differences in } \\
\text { complementary } \\
\text { feeding or } \\
\text { discontinuation of BF } \\
\text { between groups } \\
\text { Infant outcomes } \\
\text { No differences in } \\
\text { mean weight or rate of } \\
\text { growth between } \\
\text { contraceptive groups }\end{array}$ & $\begin{array}{l}\text { Strengths: } \\
\text {-Multicultural and } \\
\text { multicenter with nested } \\
\text { double blinded RCT } \\
\text { Weaknesses: } \\
\text {-No power calculation } \\
\text {-No details of method } \\
\text { switching/discontinuation } \\
\text {-No attempt to control } \\
\text { analysis for confounders }\end{array}$ & $\begin{array}{l}\text { Level II-2, Poor } \\
\text { Key Question } 1\end{array}$ \\
\hline $\begin{array}{l}\text { Abdulla et al., } 1985 \\
\text { [66] } \\
\text { Rockefeller } \\
\text { Foundation }\end{array}$ & $\begin{array}{l}\text { Cohort } \\
\text { Egypt } \\
N=20 \text { healthy women } \\
\text { after singleton, term } \\
\text { delivery (mean age } \\
29 \text { years) }\end{array}$ & $\begin{array}{l}\text { 30-39 days PP: } \\
\text { 10=LNG implant } \\
10=\text { Barriers/nothing }\end{array}$ & $\begin{array}{l}\text { Infant health (occurrence of } \\
\text { significant illnesses; serum } \\
\text { IgA, IgG, IgM) } \\
\text { Follow-up } 6 \text { months }\end{array}$ & $\begin{array}{l}\text { Infant outcomes } \\
\text { No infants had } \\
\text { significant illnesses } \\
\text { No significant } \\
\text { differences between } \\
\text { groups in infant } \\
\text { serum } \\
\text { immunoglobulins }\end{array}$ & $\begin{array}{l}\text { Weaknesses: } \\
\text {-Selection and } \\
\text { assessment procedures } \\
\text { not specified } \\
\text {-Small sample size with } \\
\text { no power calculations } \\
\text {-Percent follow-up not } \\
\text { reported }\end{array}$ & $\begin{array}{l}\text { Level II-2, Poor } \\
\text { Key Question } 1\end{array}$ \\
\hline $\begin{array}{l}\text { Shaaban et al., } 1985 \\
\text { [50] } \\
\text { Rockefeller } \\
\text { Foundation }\end{array}$ & $\begin{array}{l}\text { Cohort } \\
\text { Egypt } \\
N=150 \text { healthy, } \\
\text { multiparous, } \\
\text { BF-experienced } \\
\text { women (mean age } \\
29 \text { years) after } \\
\text { normal, term delivery }\end{array}$ & $\begin{array}{l}\text { 30 }-42 \text { days PP: } \\
50=\mathrm{LNG} \text { implant } \\
50=\mathrm{Cu} \text { T380 IUD } \\
50=\text { Barriers/nothing }\end{array}$ & $\begin{array}{l}\text { BF performance (frequency, } \\
\text { supplementation) } \\
\text { Infant growth (weight, } \\
\text { length) } \\
\text { Infant health (illness) } \\
\text { Follow-up } 6 \text { months }\end{array}$ & 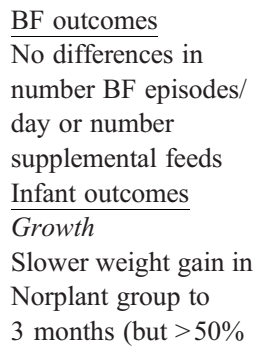 & $\begin{array}{l}\text { Weaknesses: } \\
\text {-No adjustment of for } \\
\text { possible confounders } \\
\text {-Differences at baseline } \\
\text { between groups } \\
\text {-Unclear how infant } \\
\text { morbidity was measured }\end{array}$ & $\begin{array}{l}\text { Level II-2, Poor } \\
\text { Key Question } 1\end{array}$ \\
\hline
\end{tabular}




\begin{tabular}{|c|c|c|}
\hline $\begin{array}{l}\text { Shikary et al., } 1986 \\
\text { [65] } \\
\text { WHO, Population } \\
\text { Council }\end{array}$ & $\begin{array}{l}\text { Cohort } \\
\text { India } \\
N=29 \text { women after } \\
\text { term delivery of male } \\
\text { infants, ages } 20- \\
35 \text { years }\end{array}$ & $\begin{array}{l}4 \text { weeks PP: } \\
\text { 9=POP }(\text { LNG } 30 \mathrm{mcg}) \\
\text { 10=LNG implant } \\
\text { 10=No method }\end{array}$ \\
\hline $\begin{array}{l}\text { Zacharias et al., } 1986 \\
\text { [39] } \\
\text { UpJohn, Ayerst }\end{array}$ & $\begin{array}{l}\text { Prospective cohort } \\
\text { Chile } \\
N=665 \text { women, after } \\
\text { term deliveries }\end{array}$ & $\begin{array}{l}3-6 \text { weeks PP: } \\
143=\text { LAM } \\
109=\text { Cu T IUD (presumably } \\
\text { NH) } \\
228=\text { DMPA } \\
185=\text { P O P } \quad(0.6 \mathrm{mg} \\
\text { clogestone acetate) }\end{array}$ \\
\hline $\begin{array}{l}\text { Affandi et al., } 1986 \\
\text { [67] } \\
\text { Population Council }\end{array}$ & $\begin{array}{l}\text { Cohort } \\
\text { Indonesia } \\
N=120 \text { women } \\
\text { after term, healthy } \\
\text { delivery, planning to } \\
\text { breastfeed } \\
\geq 6 \text { months }\end{array}$ & $\begin{array}{l}4-6 \text { weeks PP: } \\
60=\text { LNG implant } \\
60=\text { Copper IUD }\end{array}$ \\
\hline $\begin{array}{l}\text { McCann et al., } 1989 \\
\text { [47] } \\
\text { USAID, Family } \\
\text { Health International, } \\
\text { Wyeth }\end{array}$ & $\begin{array}{l}\text { Cohort } \\
\text { Argentina } \\
N=500 \text { healthy } \\
\text { multiparous women, } \\
\text { after term delivery }\end{array}$ & $\begin{array}{l}1 \text { week PP: } \\
250=\mathrm{LNG}(30 \mathrm{mcg}) \\
250=\mathrm{NH} \text { methods }(54 \% \\
\text { IUD) }\end{array}$ \\
\hline
\end{tabular}

Infant health (daily 4-h urine samples tested for FSH, $\mathrm{LH}$ testosterone)

Follow-up 15 weeks

BF performance (duration) Infant growth and

development (not specified) Follow-up of children to median age of 4.5 years

nfant growth (weight, length)

Follow-up 6 months

\section{BF performance}

(continuation,

Infant growth (weight, length, head circumference, percentile); no

differences at

4-6 months; slower

length increase in

Norplant group from

months 3-6 (but

$>50 \%$ percentile)

Health

No differences in

infant morbidity

Infant outcomes

No significant

differences in mean

FSH, LH and

testosterone area

under the curve

between the groups

BF outcomes

Mean duration:

17 months LAM

21 months IUD

30 DMPA

22 POP $(\mathrm{p}<.03$ for

pairwise compariso with DMPA)

nfant outcomes

Growth/development

No adverse effects of

progestogens (not specified)

Infant outcomes

Infants in LNG group

gained significantly

more weight than the

IUD group. No

differences in length

between groups

(statistical

eaknesses:

Level II-2, Fair

-Small sample size with

Key Question 1

no power calculations

-Short follow-up

Strengths:

-Survival analysis

techniques

Weaknesses:

-Measures for growth

and development not

provided

-Statistical comparisons

not performed

-No attempt to control

analysis for confounders

-Baseline differences

between groups

-Infants with "signs of inadequate nutrition" discontinued from study and not reported on

Weaknesses:

Level II-2, Poor

-Limited description of statistical analysis and no attempt to control for confounders

-Baseline differences

between groups

-Percent follow-up not

reported

not provided)

BF outcomes

Median age of

initiation of

Strength:

-Survival analysis

Level II-2, Poor

Weaknesses:

for LNG vs. 


Study design, Interventions

Study design,

Interventions

population

year,
source of support

Pharmaceuticals

with prior $\mathrm{BF}$

experience,

ages $30-35$ years

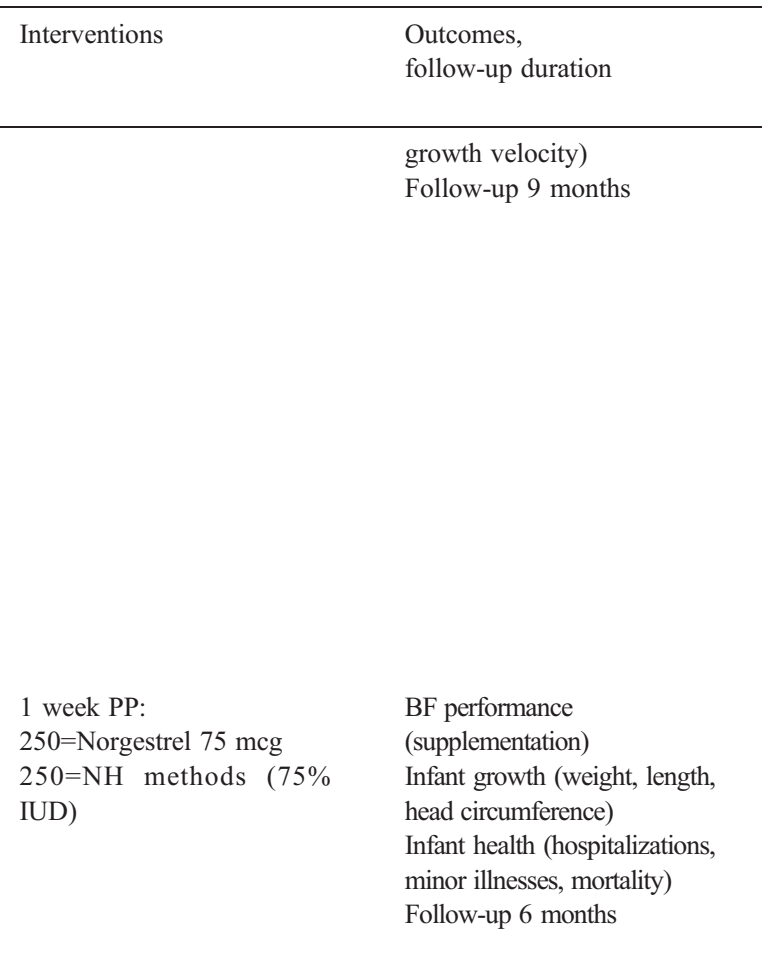

$\begin{array}{cl}\text { Moggia et al., 1991 [48] } & \text { Cohort } \\ \text { Family Health } & \text { Argentina } \\ \text { International } & N=500 \text { healthy } \\ & \text { women with } \\ & \text { experience BF, } \\ & \text { after term delivery, } \\ & \text { ages 18-35 years } \\ & (483 \text { in final analysis })\end{array}$

(483 in final analysis)

$\begin{array}{ll}\text { Shaaban, 1991 [40] } & \text { Cohort } \\ \text { WHO, Population } & \text { Egypt } \\ \text { Council, Rockefeller } & \text { Phase 1: } \\ \text { Foundation } & 360 \text { healthy women } \\ & \text { and their infants } \\ & \text { Phase 2: }\end{array}$

5th to 7 th week PP:

$120=$ LNG implant

$120=$ NET-EN injectable

Phase 2:

Results

4.6 months for $\mathrm{NH}$

users $(p<.05)$; also

significantly different

on survival analysis

$\mathrm{NH}$ users three times

more likely to

discontinue $\mathrm{BF}$

during study period

than LNG users

(22 vs. 7, p value not

reported)

Infant outcomes

No differences

between groups in

infant growth on any

measure

BF outcomes:

More frequent

supplementary feeding

in $\mathrm{NH}$ group at months

2 and $3(p<.05)$,

otherwise no

difference; no

difference in number of

women supplementing

at any time

Infant outcomes

\section{Growth}

No difference in infant

growth

Health

No differences between

groups in

hospitalizations. Minor

illnesses more common

in $\mathrm{NH}$ group (91 NH,

60 POC, $\mathrm{p}<.01) ; 3$

infant deaths in $\mathrm{NH}$

group, 0 in POP group

BF outcomes

No differences in

timing or type of

supplementation

IUD users weaned

earliest, followed by

BF performance (age of

weaning)

Infant growth (weight, arm

circumference, skinfold

thickness)
Strengths/weaknesses

Quality grading/

key question

multiparous women

-High loss to follow-up

( $55 \%$ at 9 months)

-Statistical analysis not

reported on all outcomes

of interest

-Infant health outcomes

collected but not reported

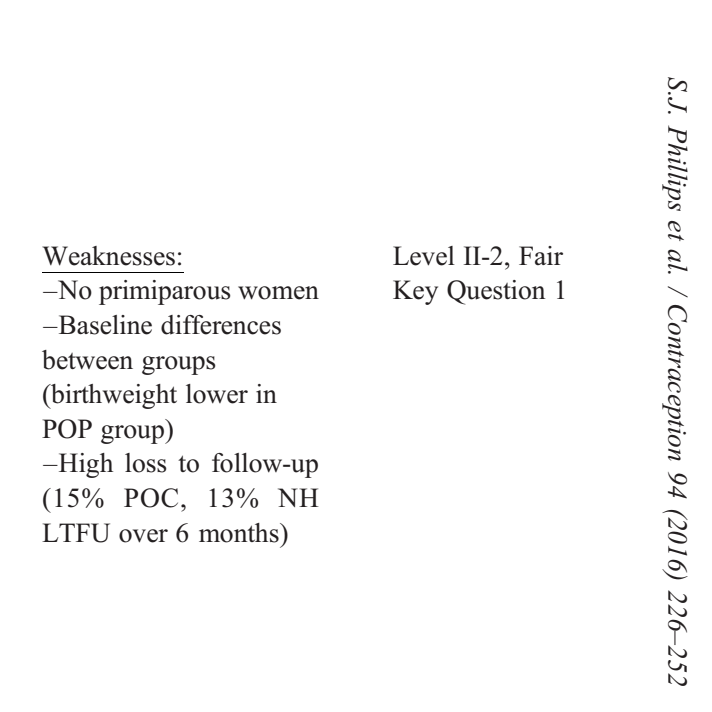

\author{
Weaknesses: \\ -Methodology poorly \\ described \\ -Baseline characteristics \\ not described \\ -Statistical analyses not
}

Level II-2, Poor

$+$


PVR and $\mathrm{Cu}-\mathrm{IUD}$,

results not discussed

$\begin{array}{lll}\text { Pardthaisong, 1992 [29] } & \text { Cohort } & \text { During lactation (any time, } \\ \text { Ford Foundation } & \text { Thailand } & 77 \% \text { initiated between } \\ \text { WHO } & N=3231 \text { infants with } & \text { months 1 and 3) } \\ \text { FHI } & \text { varying levels of } & 857=\text { DMPA only during } \\ & \text { prenatal and } & \text { lactation (not pregnancy) } \\ & \text { lactational DMPA } & 1215=\text { D M P d u ring } \\ & \text { exposure/nonexposure } & \begin{array}{l}\text { lactation, some also during } \\ \text { pregnancy }\end{array}\end{array}$

1167=No DMPA

$\begin{array}{lll}\text { WHO, } 1994[58,59] & \text { Cohort } & 6-8 \text { weeks PP: } \\ \text { WHO } & \text { Egypt, Iran, Thailand, } & 475=\mathrm{P} \mathrm{O} \mathrm{P} \mathrm{(L} \mathrm{N} \mathrm{G} \mathrm{or} \\ & \text { Kenya, Chile, Hungary } & \text { lynestrenol) } \\ N=2466 \text { married } & 541=\text { DMPA } \\ & \text { women, after term } & 121=\text { NET-EN } \\ \text { delivery and their infants } & 453=\text { LNG implant } \\ & 876=\text { NH (IUD, barriers, } \\ & \text { sterilization) }\end{array}$

Infant growth (weight, height) Length of follow-up for lactationally exposed infants unclear

BF performance (frequency, duration exclusive $\mathrm{BF}$ )

Infant growth (weight, arm circumference, skinfold thickness)

Infant health (mortality)

Infant development (age passed standard

developmental test)

Follow-up 12 months
LNG implant and NET-EN (statistics not reported) Infant outcomes Growth

No differences in

infant growth

Development

No difference in

attainment of

milestones

Infant outcomes

Growth

Relative risk for score

below $-2 \mathrm{Z}$ on growth

chart (no exposure as reference)

$1.1(0.9-1.2)$

lactational exposure

only (no prenatal

exposure); 1.2

$(1.0-1.3, \mathrm{p}<.05)$

any lactational

exposure (including

some with prenatal

exposure); RR 1.1

(0.9-1.4) for any

lactational exposure

potential confounde

BF outcomes:

Frequency and

duration of $\mathrm{BF}$

differed between

sites, but not

between

contraceptive groups

within a site

Infant outcomes

Growth

One site had larger

weight increase in

NET-EN group (6,

12 months) and

DMPA group

(3 months) compared

to $\mathrm{NH}$ group

Smaller increase in

arm circumference a reported for outcomes of interest

-Percent lost to

follow-up not reported

Strengths:

-Clear description of

methodology

-Appropriate analytical

methods

Weaknesses:

-Baseline differences

noted between DMPA

users and nonusers

-Unclear length of

follow-up

-Timing and amount of exposure to DMPA

unclear

-Unexposed may have

been using other

hormonal methods

Strengths:

-Large cohort,

multicultural and

multicenter

-Standardized assessment

of development

- Confounders assessed and controlled for in analysis

Weaknesses:

-Large differences

between sites for BF performance and infant outcomes

-Percent lost to follow-up not reported
Level II-2, Poor

Key Question 1 


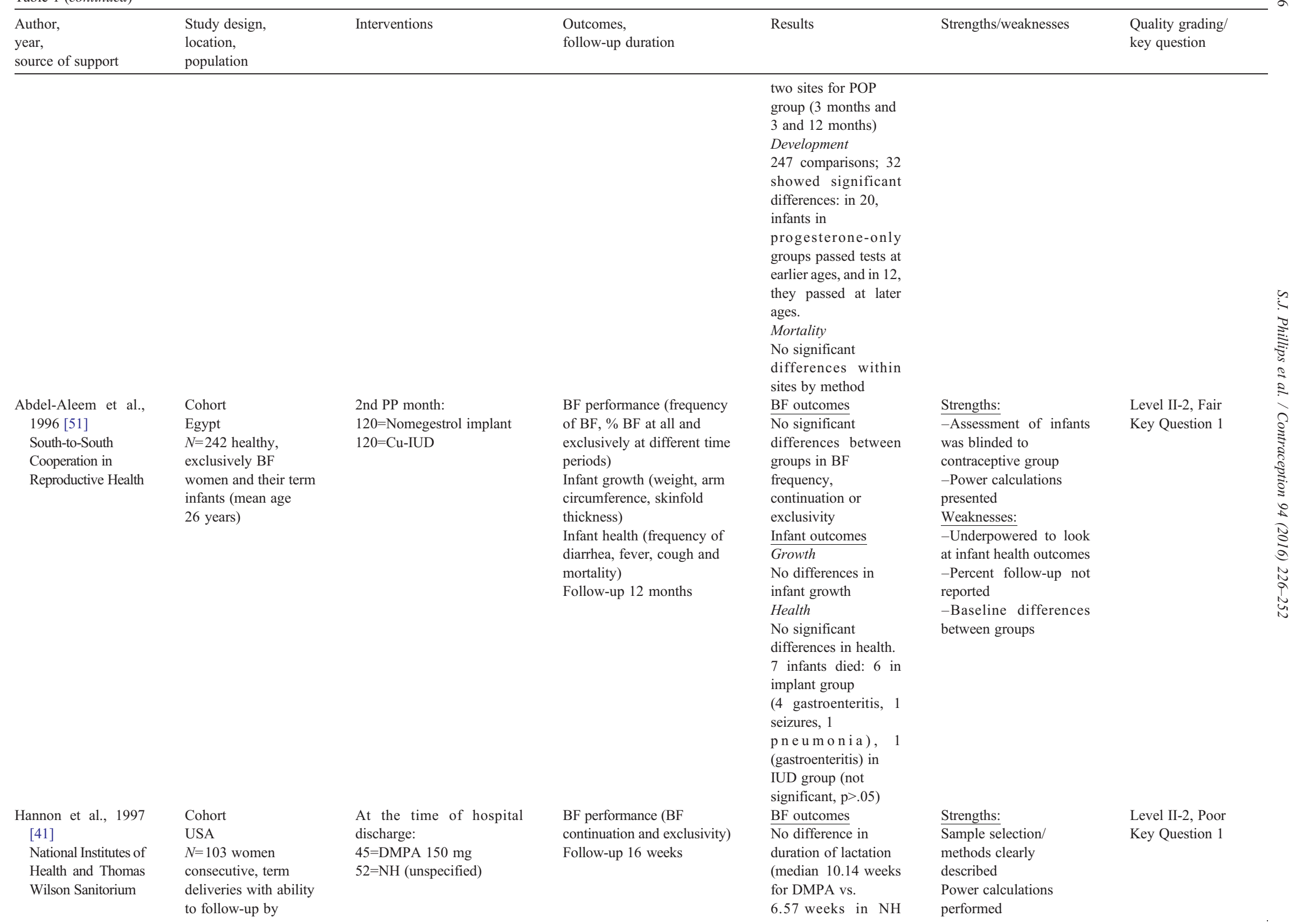




\begin{tabular}{|c|c|}
\hline $\begin{array}{c}\text { Diaz et al., } 1997 \text { [60] } \\
\text { WHO, Population } \\
\text { council, CONRAD }\end{array}$ & $\begin{array}{l}\text { Cohort with historical } \\
\text { control } \\
\text { Chile } \\
N=662 \text { cohabitating } \\
\text { parous ( } 1-3 \text { ) women } \\
\text { after term delivery, } \\
\text { ages } 18-38 \text { years }\end{array}$ \\
\hline $\begin{array}{l}\text { Lawrie et al., } 1998 \\
\text { [35] } \\
\text { Schering Ltd, Iris } \\
\text { Ellen Hodges Trust } \\
\text { of the University of } \\
\text { the Witwatersrand, } \\
\text { South African } \\
\text { Medical Research } \\
\text { Council, } \\
\text { South African } \\
\text { Institute for Medical } \\
\text { Research }\end{array}$ & $\begin{array}{l}\text { RCT } \\
\text { South Africa } \\
N=166 \text { immediate PP } \\
\text { women ages }>19 \text { years }\end{array}$ \\
\hline $\begin{array}{l}\text { Coutinho et al., } 1999 \\
\text { [64] } \\
\text { Rockefeller } \\
\text { Foundation }\end{array}$ & $\begin{array}{l}\text { Prospective cohort } \\
\text { Brazil } \\
N=135 \text { women, } \\
18-35 \text { years old after } \\
\text { term health delivery } \\
\text { planning to } \\
\text { breastfeed for } \\
6 \text { months and their } \\
\text { infants }\end{array}$ \\
\hline
\end{tabular}

Diaz et al., 1999 [28]
Population Council

Newly identified
$57 \pm 3$ days PP:

117=POP (lynestrenol)

$187=$ PVR

$120=$ LNG implant

$122=$ Copper IUD

$236=\mathrm{NH}(\mathrm{LAM})$

$<48$ h PP:

$85=$ NET-EN

$84=$ Placebo

All women additionally

used an NH method

6 weeks PP:

66=Elcometrine implant

$69=\mathrm{Cu}-\mathrm{IUD}$

(a)

BF performance (duration of any and exclusive BF)

Infant growth (weight) Follow-up 6 months

$\mathrm{BF}$ performance (duration of any $\mathrm{BF}$, exclusive or partial) Maternal depression (not reported here)
BF outcomes

No difference

between groups for

mean and total

duration of $\mathrm{BF}$

Infant outcomes

No differences in

growth between

groups

BF outcomes

difference

between groups in

continuation rates at

6 or 12 weeks

$\mathrm{BF}$ performance (any BF at follow-up time points)

Infant growth (weight, arm circumference, skinfold

thickness)

Infant development (age meeting standard milestones,

using developmental tests)

Follow-up 12 months

BF outcom

Higher rates in

implant group

(95-76\%) vs. IUD

$(84-57 \%)$ at 3 ,

differences at 9 ,

12 months

Infant outcomes
6 months $(\mathrm{p}<.05)$, no

Growth

No differences

between groups

Development

No differences in age

met developmental

milestones

$57 \pm 3$ days PP

29=LNG implant

$51=\mathrm{Cu}-\mathrm{IUD}$

$28=$ PVR (results not

reported here)
BF performance (any or

exclusive BF up to 6 months, no milk supplementation at

12 months)

Infant growth (weight)
BF outcomes

93\% LNG, 86\% IUD

(no difference);

Fully BF month 12 . users, $\mathrm{p}=.19$ )

No difference in time

to supplementatio

with formula

Weaknesses:

-Limited duration of follow-up

-No infant outcomes

-Baseline differences

between groups (DMPA

younger, unmarried)

Weaknesses:

Historical control

Level II-2, Fair

Key Question 1

Strengths:

-Clear description of

Level I,

methods

Key Question 1

-Enrolled women

regardless of past/current

BF experience

Weaknesses:

-Small sample size

-BF outcomes were

secondary

\section{Strengths:}

-Power calculation

performed

-Standardized outcomes

used and described

Weaknesses:

-No control for potential

confounders

Fully BF month 6:

Strengths:

Level II-2, Fair

-Clearly described

Key Question 1

Level II-2, Fair

Key Question 1

Weaknesses:

to breastfeed

-BF/Infant outcomes 


Interventions

Outcomes,

follow-up duration

Results

Strengths/weaknesses

Quality grading/

Quality grading
key question

source of support

populatio

Follow-up minimum

12 months

$\begin{array}{cl}\text { Bjarnadóttir et al., } & \text { Cohort } \\ 2001[46] & \text { Iceland } \\ \text { Organon } & N=83 \text { multiparous } \\ & \text { women with prior } \\ & \text { experience BF, after } \\ & \text { term delivery, ages }\end{array}$

28-56 days PP:

$42=$ Desogestrel $75 \mathrm{mg}$

$41=\mathrm{Cu} 375$ IUD

BF performance (any BF)

Infant growth (length,

Infant health (intercurrent

illness, hospitalizations)

$18-40$ years

Follow-up 2.5 years

Baheiraei et al., 2001
[68]

Prospective cohort

Iran

Not stated
$N=140$ women, after healthy term delivery
6 weeks PP:

51 =Progestogen-only

(DMPA; POP)

$89=\mathrm{NH}$ (IUD, condom,

sterilization)
Infant growth (weight,

length, head circumference)

Follow-up 26 weeks
$4 \%$ LNG, $10 \%$ IUD

(no difference)

Duration of lactation

15 months LNG,

14 months IUD (no

difference)

Infant outcomes

Growth

No difference

between LNG and

$\mathrm{Cu}$-IUD groups at

month 1,6 or 12

BF outcomes:

No difference in BF

continuation at cycle

4 (5-6 months PP),

but at end cycle 7

(8-9 months PP),

$78 \%$ in desogestrel

compared with $59 \%$

in IUD group were

still BF (statistics not

reported)

Infant outcomes

Growth

No differences

Health

Temporary breast

enlargement in 2

infants, increased

sweating in 1 infant in

desogestrel group; no

occurrences in IUD

group. No other

differences in health

were secondary

-Length of follow-up

unclear

-Loss to follow-up not specified

nfant outcomes

Growth

No differences in

weight or length at

any time; mean head

circumference change

$1.42 \mathrm{~cm}$

(progestogen-only)

vs. $1.19(\mathrm{NH})$ at $10-$

13 weeks $(\mathrm{p}<.05)$. No
Strengths:

-Power calculations

provided

-Clearly described

methods

-Long-term follow-up of

exposed infants

Weaknesses:

-Wide variation in

timing of contraceptive

initiation

Weaknesses:

-Contraceptive use

switching or

formulations are not

stated

-Separate estimates for different methods not presented

-Percent lost to

follow-up not reported
Level II-2, Fair

Key Question 1 


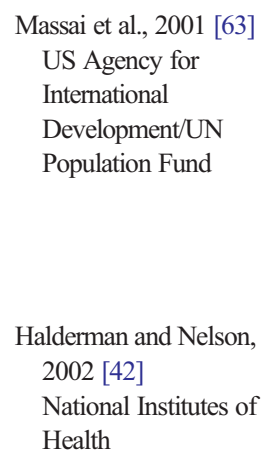

Development/UN

Population Fund

Halderman and Nelson 2002 [42]

National Institutes of

Health

\section{Prospective cohort}

Chile

$N=200$ cohabitating

women, after term

delivery; ages $18-38$ years

\section{Cohort}

USA

$N=319$ women, primarily Hispanic, ages 16-49 years

\section{Prospective cohort}

from 2 previous

studies

Chile

$N=442$ cohabitating parous (1-3) women after term delivery, ages $18-35$ years
55-60 days PP:

$100=$ Nesterone implant

$100=$ Copper IUD

ctation performance nfant growth (weight gain)

Follow-up 1 year

Prior to discharge from hospital:

102 $=$ DMPA

$79=\mathrm{LNG}$ implant or $\mathrm{POP}$ $138=\mathrm{NH}$

$55 \pm 3$ days PP

$220=$ LNG implant

$222=$ Copper IUD

BF performance (initiation, continuation, supplementation)

Follow-up 6 weeks

BF performance (duration)

Infant growth (weight,

height) and health differences at other

time points

BF outcomes

No difference in $\mathrm{BF}$

episodes per day or

length of $\mathrm{BF}$ ( 273 days

implant vs. 263 IUD)

Infant outcomes

Growth

No differences

BF outcomes

No difference in $\mathrm{BF}$

initiation

Any BF at 4 weeks:

$83.1 \% \mathrm{NH}, 76.7 \%$

$\mathrm{POC}(\mathrm{p}=.022)$

Any $\mathrm{BF}$ at 2 ,

6 weeks: No

difference

Exclusivity,

supplementation: No

difference at any time

BF outcomes

Follow-up 6 years

No differences in

mean and tota

duration; at $12 \mathrm{th}$
Strengths:

Level II-2, Fair

contraceptive

switching and

discontinuation

Weaknesses:

-High discontinuation

(17\% in NES group and

$22 \%$ in IUD group)

Weaknesses:

Level II-2, Poor

-Short follow-up

-No infant outcomes

-Aggregate data for

methods other than

DMPA

-Differences between

groups at baseline

(DMPA younger, les parous, less experienced with $\mathrm{BF}$ )

\section{Strengths} month, LNG group

had fewer fully BF

Infant outcomes

Growth

No differences

Health

Higher incidence of

respiratory infection

(colds, bronchitis;

44.3 vs. $37.7 / 100$

infant months,

$\mathrm{p}<.0001)$ and skin

conditions (diaper,

allergic and bacterial

dermatitis, prurigo) in

LNG group. Higher

incidence of

urogenital disease

(0.4 vs. 0.2$)$ and

psychomotor

pairment (23 vs.
-Information on

contraceptive switching

and discontinuation

-Long-term follow-up

-Adjusted for potential

confounders

-Power calculation for

infant growth

-Blinded assessment of

health

-Verification with

hospital records

Weaknesses:

-High loss to f/u over

time $(14 \%$ of implant

group and $21 \%$ of IUD

group)

-No power calculations

for health outcomes

-Confounders for skin

disease not assessed

-Data from infants from

different time periods

Pollution levels in

Santiago may limit 


Study design, Interventions

Study design,

Interventions

year,
source of support

populatio

Outcomes,

follow-up duration

Results

Strengths/weaknesses

Quality grading/

key question

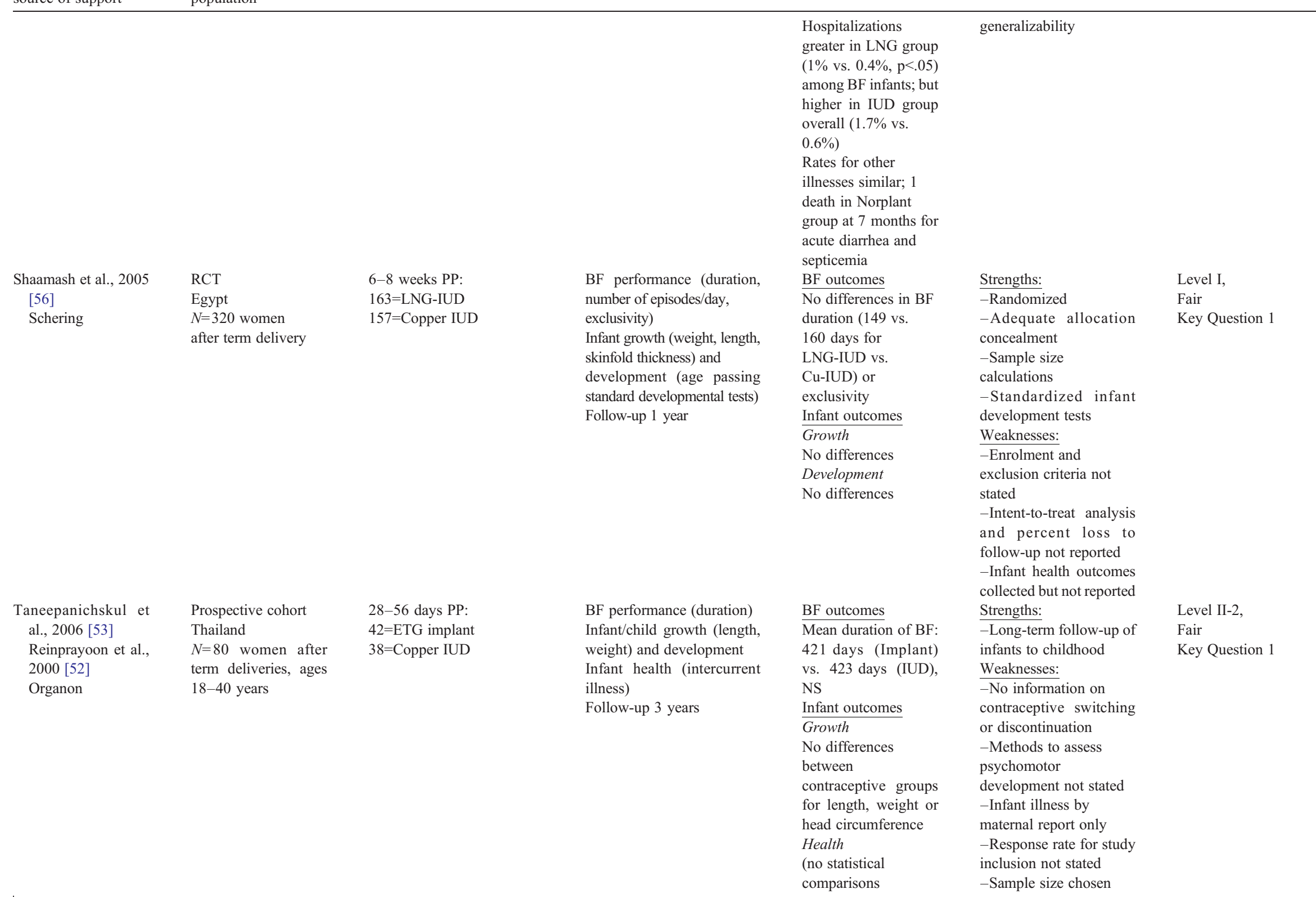

pent/child growth (length,

weight) and development

Infant health (intercurren

illness) ospitalizations

Rates for other

death in Norplan

group at 7 months for

cute diarrhea an

F outcom

60 days for

Growth

Mean duration of $\mathrm{BF}$ :

421 days (Implant)

NS

atcomes

No difference

(no statistical

-Sample size chosen 


Brito et al., $2009[20]$
FAPESP
CNPq
Newly identified

RCT (open label)

Brazil

$N=40$ women with

35 years

Chen et al., $2011[22]$
Anonymous
foundation
Newly identified

United States

$N=96$ women

interested in PP IUD
$\mathrm{BMI}<30$, ages $18-$
24-48 h following delivery: $20=$ Etonorgestrel implant (ETG)

6 weeks PP:

$20=150 \mathrm{mg}$ DMPA reported)

$\mathrm{BF}$ performance (duration of

RCT (open label) Immediate postplacental:
$50=$ LNG-IUD

6-8 weeks PP (delayed)

$46=$ LNG-IUD
BF outcomes:

Initiation

BF performance (initiation, duration)

Follow-up 6 months
$10 / 42$ implant infants

reported skin/

appendages disorders

vs. 6/38 IUD infants;

17/42 implant infants

reported respiratory

system disorders vs.

10/38 IUD infants; 3

42 implant infant reported GI disorders vs. 5/38 IUD infants, 2/42 implant infants

reported neonatal/ infancy disorders vs. 2/38 IUD infants

No differences

reported in adverse

events or

psychomotor

development (>60\%

infants in both group

had resp. disorders,

and $>30 \%$ in both

groups had skin

disorders)

BF outcomes

No difference in

exclusive $\mathrm{BF}$ between

groups at 6 weeks or

12 weeks: (6 weeks

95\% ETG, $85 \%$

DMPA; 12 weeks

85\% ETG, 75\%

DMPA)

Infant outcomes

Growth

No differences at 6 o

12 weeks

based on WHO

recommendations for

toxicology, not for BF

or health outcomes

Strengths:

-Randomization

methods appropriate

-Allocation concealment

appropriate

-Methods clearly

described

Weaknesses:

-Short follow-up

-Small sample size with

no power calculations

Strengths:

-Randomization

methods appropriate

$32 / 50$ (postplacental)

$27 / 46$ (delayed) $\mathrm{p}=.59$

Duration

-Allocation concealment

5 week

appropriate

-Methods clearly

described

Weaknesses

8.5 weeks (delayed)

$\mathrm{p}=.06$

Any BF at 6 months 


\begin{tabular}{|c|c|c|c|c|c|c|}
\hline $\begin{array}{l}\text { Author, } \\
\text { year, } \\
\text { source of support }\end{array}$ & $\begin{array}{l}\text { Study design, } \\
\text { location, } \\
\text { population }\end{array}$ & Interventions & $\begin{array}{l}\text { Outcomes, } \\
\text { follow-up duration }\end{array}$ & Results & Strengths/weaknesses & $\begin{array}{l}\text { Quality grading/ } \\
\text { key question }\end{array}$ \\
\hline & & & & $\begin{array}{l}3 / 50 \text { postplacental, } \\
11 / 46 \text { delayed } \mathrm{p}=.02 \\
\text { Exchisive } B F \text { at } 6 \text { months } \\
1 / 50 \text { postplacental, } 6 \text { / } \\
46 \text { delayed } \mathrm{p}=.05\end{array}$ & $\begin{array}{l}\text { prior to LNG-IUD } \\
\text { placement } \\
\text {-Short follow-up } \\
\text {-Very low rates BF in } \\
\text { both groups may limit } \\
\text { generalizability }\end{array}$ & \\
\hline $\begin{array}{l}\text { Gurtcheff et al., } 2011 \\
\text { [23] } \\
\text { National Center for } \\
\text { Research Resources } \\
\text { Newly identified }\end{array}$ & $\begin{array}{l}\text { RCT (open label) } \\
\text { United States } \\
N=69 \text { peripartum } \\
\text { women desiring ETG } \\
\text { implant }\end{array}$ & $\begin{array}{l}\text { Insertion at } 1-3 \text { days PP: } \\
35=\text { ETG implant (early) } \\
4-8 \text { weeks PP } \\
34=\text { ETG implant (standard) }\end{array}$ & $\begin{array}{l}\text { BF performance (time to } \\
\text { lactogenesis stage II, } \\
\text { lactation failure, formula } \\
\text { supplementation) } \\
\text { Follow-up } 6 \text { months }\end{array}$ & $\begin{array}{l}\frac{\text { BF outcomes }}{\text { Mean time to }} \\
\text { lactogenesis stage II } \\
64 \mathrm{~h} \text { (early); } 65 \mathrm{~h} \\
\text { (standard) } \\
\text { Lactation failure } \\
1 / 34 \text { early, } 0 / 35 \\
\text { standard, risk } \\
\text { difference } 0.03 \text { (early } \\
\text { vs. standard) } \\
\text { Formula } \\
\text { supplementation } \\
\text { No difference } \\
\text { between groups at } \\
2 \text { weeks, } 4-8 \text { weeks, } \\
3 \text { months or } 6 \text { months }\end{array}$ & $\begin{array}{l}\text { Strengths: } \\
\text {-Randomization } \\
\text { methods appropriate } \\
\text {-Allocation concealment } \\
\text { appropriate } \\
\text {-Methods clearly } \\
\text { described } \\
\text {-Power calculations } \\
\text { presented } \\
\text { Weaknesses: } \\
-32 \%(11 / 34) \text { women } \\
\text { randomized to standard } \\
\text { insertion did not receive } \\
\text { implant; as a result } \\
\text { analyses are per-protocol }\end{array}$ & $\begin{array}{l}\text { Level I, Fair } \\
\text { Key Question } 2\end{array}$ \\
\hline $\begin{array}{l}\text { Costa et al., } 2012 \text { [26] } \\
\text { FAPESP } \\
\text { Newly identified }\end{array}$ & $\begin{array}{l}\text { Cohort } \\
\text { Brazil } \\
N=82 \text { PP women }\end{array}$ & $\begin{array}{l}6 \text { weeks PP: } \\
28=\text { POCs (DMPA, POP, } \\
\text { LNG-IUD) } \\
\text { 54=NH (Barrier, LAM, TL, } \\
\text { Cu-IUD) }\end{array}$ & $\begin{array}{l}\mathrm{BF} \text { performance (exclusive } \\
\text { and total BF duration) } \\
\text { Follow-up } 6 \text { months }\end{array}$ & $\begin{array}{l}\text { BF outcomes } \\
\text { Exclusive: } \\
\text { Mean duration } \\
137 \text { days } \mathrm{NH}, \\
113 \text { days } \mathrm{POC}(\mathrm{p}=.143) \\
\text { Total (any } B F): \\
183 \text { days } \mathrm{NH}, 183 \text { days } \\
\mathrm{POC}(\mathrm{p}=.383)\end{array}$ & $\begin{array}{l}\text { Strengths: } \\
\text {-Included primiparas } \\
\text {-Clear description of methods } \\
\text { Weaknesses: } \\
\text {-No separate analysis of } \\
\text { different POCs } \\
\text {-BF outcomes were } \\
\text { secondary outcomes }\end{array}$ & $\begin{array}{l}\text { Level II-2, Fair } \\
\text { Key Question } 1\end{array}$ \\
\hline $\begin{array}{l}\text { Espey et al., } 2012 \\
{[21]} \\
\text { ACOG contraceptive } \\
\text { grant and University } \\
\text { of New Mexico } \\
\text { Newly identified }\end{array}$ & $\begin{array}{l}\text { RCT (double blinded) } \\
\text { US } \\
N=127 \text { women ages } \\
15-45 \text { years, planning } \\
\text { to BF and use oral } \\
\text { contraceptives }\end{array}$ & $\begin{array}{l}2 \text { weeks PP: } \\
64=\mathrm{COC} \text { ( } 0.35 \mathrm{mg} \text { ethinyl } \\
\text { estradiol, } 1 \mathrm{mg} \text { norethindrone) } \\
65=\mathrm{POP} \text { (norethindrone } \\
0.35 \mathrm{mg} \text { norethindrone) }\end{array}$ & $\begin{array}{l}\text { BF performance (BF } \\
\text { continuation at } 8 \text { weeks, } \\
6 \text { months; supplementation } \\
\text { at } 8 \text { weeks) } \\
\text { Infant growth (weight, } \\
\text { length, head circumference) } \\
\text { Follow-up } 6 \text { months for BF } \\
\text { outcomes, } 2 \text { months for } \\
\text { infant outcomes }\end{array}$ & $\begin{array}{l}\text { BF outcomes: } \\
\text { No difference in } \\
\text { continuation at } \\
8 \text { weeks ( } 64 \% \text { COC, } \\
63.5 \% \text { POP) or over } \\
6 \text { months (survival } \\
\text { analysis); no } \\
\text { difference in } \\
\text { supplementation at } \\
8 \text { weeks (percents not } \\
\text { reported) } \\
\text { Infant outcomes } \\
\text { Growth } \\
\text { No difference through } \\
8 \text { weeks }\end{array}$ & $\begin{array}{l}\text { Strengths: } \\
\text {-Included primiparas } \\
\text {-Randomization } \\
\text { methods appropriate } \\
\text {-Allocation concealment } \\
\text { appropriate } \\
\text {-Methods clearly } \\
\text { described } \\
\text {-Double-blinded } \\
\text {-Minimal method } \\
\text { switching } \\
\text {-Loss to follow-up } \\
\text { similar between groups } \\
\text { Weaknesses } \\
\text {-Small sample size }\end{array}$ & $\begin{array}{l}\text { Level I, Fair } \\
\text { Key Question } 1\end{array}$ \\
\hline
\end{tabular}

RCT (open label) United States

plant (early)

8 weeks PP

National Center for Research Resources implan

$28=$ POCs (DMPA, POP, LNG-IUD)

54=NH (Barrier, LAM, TL Cu-IUD)
$\mathrm{BF}$ performance (time to actogenesis stage II,

$1 / 34$ early, $0 / 35$

Formula

No difference

37 days $\mathrm{NH}$

183 days NH, 183 days

POC $(\mathrm{p}=.383)$

No difference in

continuation

-Small sample size 


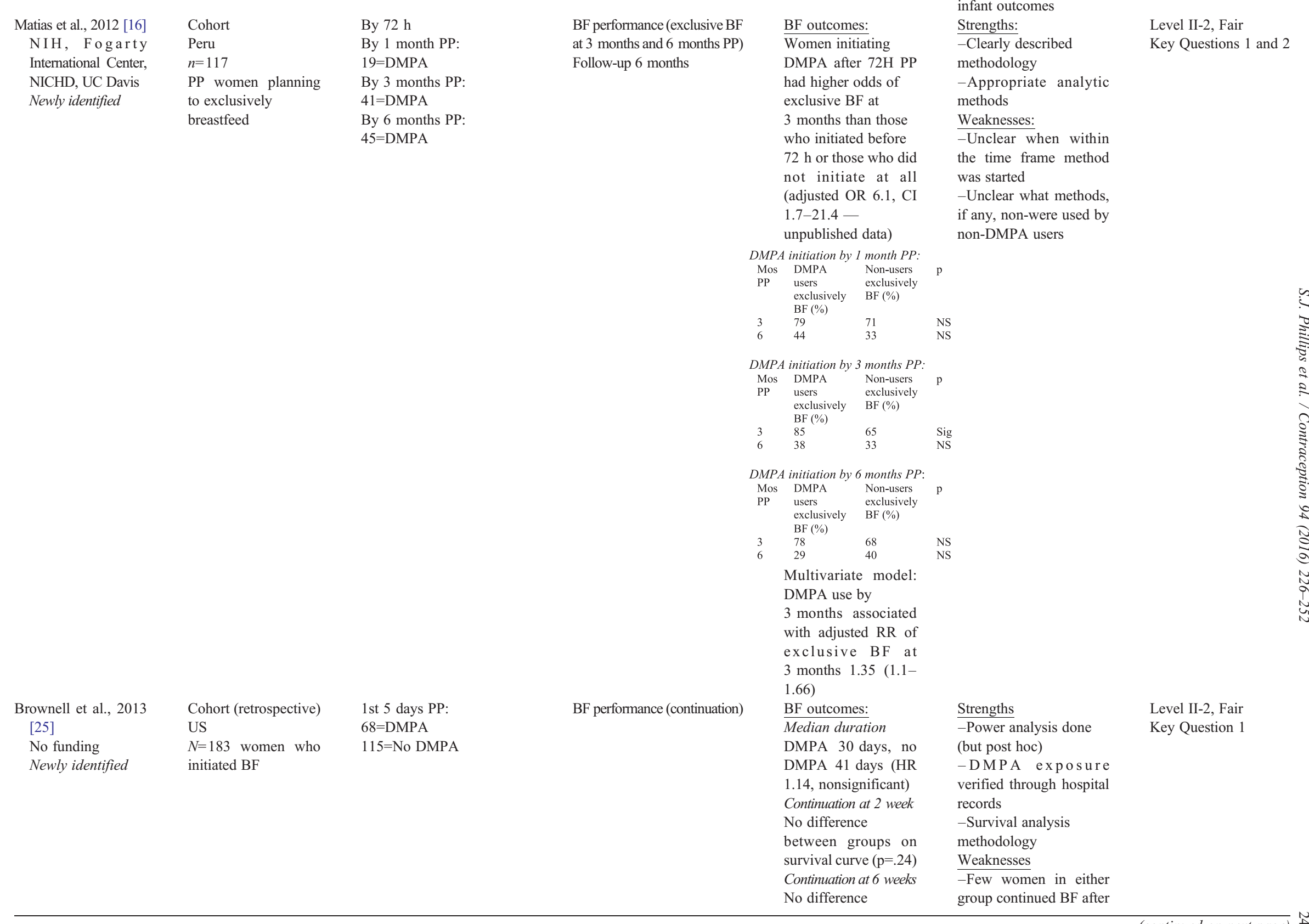




\begin{tabular}{|c|c|c|c|c|c|c|}
\hline $\begin{array}{l}\text { Author, } \\
\text { year, } \\
\text { source of support }\end{array}$ & $\begin{array}{l}\text { Study design, } \\
\text { location, } \\
\text { population }\end{array}$ & Interventions & $\begin{array}{l}\text { Outcomes, } \\
\text { follow-up duration }\end{array}$ & Results & Strengths/weaknesses & $\begin{array}{l}\text { Quality grading/ } \\
\text { key question }\end{array}$ \\
\hline $\begin{array}{l}\text { Bahamondes et al., } \\
2013 \text { [24] } \\
\text { FAPESP, Conselho } \\
\text { Nacional de Pesquisa } \\
\text { Newly identified }\end{array}$ & $\begin{array}{l}\text { Prospective cohort } \\
\text { Brazil } \\
N=40 \text { multiparous } \\
\text { women with prior BF } \\
\text { experience }\end{array}$ & $\begin{array}{l}\text { Day } 42 \text { PP: } \\
10=\text { COC } \\
10=\text { ETG implant } \\
10=\text { LNG-IUD } \\
10=\text { Cu-IUD }\end{array}$ & $\begin{array}{l}\text { BF performance (duration, } \\
\text { number of episodes/day) } \\
\text { Infant growth (weight, } \\
\text { height, tibial length) } \\
\text { Infant salivary deuterium } \\
\text { (data not presented) } \\
\text { Follow-up } 3 \text { weeks (growth } \\
\text { outcomes); } 6 \text { months (BF } \\
\text { outcomes) }\end{array}$ & $\begin{array}{l}\text { between groups (HR } \\
1.22, \mathrm{p}=.42 \text { ) } \\
\text { Insufficient events } \\
\text { after } 6 \text { weeks to draw } \\
\text { conclusions } \\
\text { BF outcomes: } \\
\text { No significant difference } \\
\text { among groups in } \\
\text { continued BF at } 6 \\
\text { months (data not shown) } \\
\text { Significantly more BF } \\
\text { episodes day } 4 \text { of } \\
\text { study in ETG vs. } \\
\text { Cu-IUD; otherwise no } \\
\text { difference (data not } \\
\text { shown) } \\
\text { Infant outcomes } \\
\text { No significant } \\
\text { difference in weight } \\
\text { or height change from } \\
\text { days } 42-63 \text {; significant } \\
\text { difference in increase in } \\
\text { tibial length (0.6 cm vs. } \\
1.3 \mathrm{~cm} \text { ) in ETG vs. } \\
\text { Cu-IUD, otherwise no } \\
\text { significant difference }\end{array}$ & $\begin{array}{l}6 \text { weeks } \\
\text {-Unclear if women in } \\
\text { non-DMPA group were } \\
\text { exposed after } 5 \text { days to } \\
\text { either DMPA or to other } \\
\text { contraceptives } \\
\text { Strengths: } \\
\text {-Frequent data } \\
\text { collection } \\
\text { - Clearly described } \\
\text { methodology } \\
\text { Weaknesses: } \\
- \text { Short follow-up (other } \\
\text { than BF duration measure) } \\
\text {-Small sample size with no } \\
\text { power calculations reported }\end{array}$ & $\begin{array}{l}\text { Level II-2, Poor } \\
\text { Key Question } 1\end{array}$ \\
\hline $\begin{array}{l}\text { Singhal et al., } 2014 \\
\text { [27] } \\
\text { Not stated } \\
\text { Newly identified }\end{array}$ & $\begin{array}{l}\text { Prospective cohort } \\
\text { India } \\
N=250 \text { women who } \\
\text { initiated BF }\end{array}$ & $\begin{array}{l}\text { Days } 3-10 \text { PP: } \\
150=\text { DMPA (only } 100 \text { with } \\
\text { full follow-up) } \\
100=\mathrm{NH}\end{array}$ & $\begin{array}{l}\text { BF performance (duration, } \\
\text { number of episodes/day) } \\
\text { Infant growth (weight, } \\
\text { length) } \\
\text { Infant health (episodes of } \\
\text { diarrhea, URI, fever, rash) } \\
\text { Follow-up } 6 \text { months }\end{array}$ & $\begin{array}{l}\text { BF outcomes: } \\
\text { No significant } \\
\text { difference between } \\
\text { groups in frequency/ } \\
\text { continued BF at } \\
6 \text { weeks or } 3 \text { or } \\
6 \text { months } \\
\text { Infant outcomes } \\
\text { No significant } \\
\text { differences between } \\
\text { groups in weight or } \\
\text { length gain at any } \\
\text { time point } \\
\text { No significant } \\
\text { differences between } \\
\text { groups in illnesses }\end{array}$ & $\begin{array}{l}\frac{\text { Strengths }}{\text {-Included primiparas }} \\
\text {-DMPA exposure verified } \\
\text { Weaknesses } \\
\text {-High LTFU and no } \\
\text { information provided on } \\
\text { DMPA users who failed } \\
\text { to provide } 6 \text { months } \\
\text { follow-up (50/150) } \\
\text {-Comparison group } \\
\text { poorly described; unclear } \\
\text { which NH methods were } \\
\text { used } \\
\text {-No power calculations } \\
\text { reported }\end{array}$ & $\begin{array}{l}\text { Level II-2, Poor } \\
\text { Key Question } 1\end{array}$ \\
\hline
\end{tabular}

Abbreviations: ACOG: American College of Obstetrics and Gynecology; BF: breastfeeding; CEBRE: Center for the Study of Reproductive Biology; FAPESP: Fundação de Amparo à Pesquisa do Estado de São Paulo; NET: norethisterone; NH: nonhormonal; PP: postpartum; TL: tubal ligation. 
include progestogen-only pills (POPs), progestogen and progesterone implants, injectables, progesterone rings and progestogen-releasing intrauterine devices (IUDs). They are highly effective when used as directed [6].

The use of progestogen-only methods of contraception [progestogen-only contraceptives (POCs)] during the period of lactation has raised concerns for negative effects [7]. Progestogens could interfere with lactogenesis, especially immediately postpartum [8], and have been shown to be transferred to breast milk [9]. Animal data suggest that progesterone receptors are common in the developing rat forebrain [10]. It is therefore possible that POCs may affect infant health or development [11]. The large loading dose of progestogens found in the injectable depot medroxyprogesterone acetate (DMPA) has been particularly called into question [7].

This systematic review was conducted for the WHO's Medical Eligibility Criteria for Contraceptive Use (MEC) [12] and examines the effects of POCs on outcomes such as breastfeeding performance and infant growth, development and health. It updates a previous review from 2010 [13].

\section{Methods}

We followed PRISMA (Preferred Reporting Items for Systematic Reviews and Meta-Analyses) guidelines for the conduct of systematic reviews. [14]

\subsection{Key questions}

We identified two key questions of interest: (1) Among breastfeeding women and their infants, was the use of POCS associated with a difference in breastfeeding or infant outcomes, compared with nonuse of POCs? (2) Among breastfeeding women and their infants, was initiation of POCs before 6 weeks postpartum associated with a difference in breastfeeding or infant outcomes, compared initiation of POCs at 6 weeks or later?

\subsection{Search strategy}

We searched PubMed for relevant articles in all languages published or in press from database inception through December 15, 2014 (see Appendix I). We searched reference lists of relevant articles for additional citations of interest. We did not consider unpublished studies, abstracts or dissertations. We had previously contacted one author for clarification regarding allocation between treatment groups [15] and contacted another for clarification of method of analysis and measures of association of interest [16].

\subsection{Study selection}

We included primary reports of studies of breastfeeding women who received POCs (oral, injectable, implantable or hormonal IUDs), as well as progesterone pellets. Studies assessing progesterone vaginal rings (PVRs) [17] were excluded as they were reviewed separately [18]. The main outcomes were breastfeeding performance and infant health. Studies that reported solely on self-perceived ability to breastfeed (without any reporting on duration of breastfeeding), breastfeeding episodes, milk composition or milk quantity were excluded. Studies that did not specify when contraceptives were initiated were also excluded. Studies that compared use of POCs with use of another type of hormonal contraceptive were considered indirect evidence. We included trials, cohort and case-control studies and excluded cross-sectional and noncomparative studies.

\subsection{Study quality assessment}

Two authors assessed the quality of each study (SP and NT) using the United States Preventive Services Task Force evidence grading system [19].

\subsection{Data synthesis}

We used a standard data abstraction template to systematically assess and summarize the evidence. Because many studies and recommendations separate results by the use of contraception before and after 6 weeks postpartum, we structured this report similarly. Summary odds ratios were not calculated, given the heterogeneity of interventions, results and nonquantifiable outcomes reported.

\section{Results}

The literature search yielded 848 articles; 771 were excluded on title and abstract review and 28 were excluded after full-text review, leaving 49 reports meeting inclusion criteria. Since this review was last updated in 2008 [13], four new randomized controlled trials (RCTs) [20-23] and five new observational studies were published [16,24-27], and an additional five observational studies that were not included in the 2008 review were identified [28-32], for a total of eight reports of RCTs and 41 reports of nonrandomized clinical trials or observational studies for review (Table 1). These 49 articles reported on 47 different studies investigating the use of POCs in breastfeeding women and reported clinically relevant outcomes of infant growth, health or breastfeeding performance.

Results for Key Question One, then for Key Question Two, are presented by study design and by time of contraceptive initiation: less than 6 weeks or greater than or equal to 6 weeks postpartum. Newly identified studies are presented first, followed by a brief summary of findings from the previous review. Nonrandomized clinical trials are presented together with observational data.

\subsection{Key Question One, initiation at less than 6 weeks postpartum: Lactation performance}

\subsubsection{Randomized clinical trials}

Four RCTs [21,33-35] investigated POC initiation within 6 weeks postpartum. A new RCT provides indirect evidence: 
this trial randomized 127 women planning to breastfeed to either POPs or combined oral contraceptives (COCs), started 2 weeks postpartum [21]. No difference was noted between groups in breastfeeding continuation or supplementation over 6 months.

Three RCTs were included in the previous review. In one, fewer levonorgestrel (LNG) IUD users were breastfeeding than copper $(\mathrm{Cu})$ IUD users at 75 days; this difference disappeared at 6 months [34]. Mean duration of breastfeeding was similar. Another investigating the use of norethindrone compared with placebo found no difference between groups in breastfeeding initiation [33]. A third found no difference in breastfeeding outcomes over 12 weeks between women who received injectable norethisterone enanthate (NET-EN) or placebo [35].

\subsubsection{Nonrandomized clinical trials and observational studies}

3.1.2.1. Injectables. In 11 nonrandomized clinical trials and observational studies, four of which are newly included since the last review [16,25,27,31], progestogen-only injectables (POIs) (either DMPA or NET-EN) were initiated in the first 6 weeks postpartum; most of these found either no effect on breastfeeding outcomes or improved outcomes among DMPA users. In one new prospective cohort study, women initiated DMPA or a nonhormonal method postpartum; no difference in breastfeeding frequency or continuation was observed at 6 weeks or at 3 or 6 months [27]. A second prospective cohort study found that women who initiated DMPA after $72 \mathrm{~h}$ were more likely to exclusively breastfeed at 3 months than those who either did not initiate or initiated early [16]. No differences emerged in exclusive breastfeeding to 6 months for those who did not initiate DMPA compared with those who initiated by 3 or 6 months. A third retrospective cohort study found no significant differences in duration or continuation of breastfeeding through 6 weeks between women who initiated DMPA before 5 days postpartum compared with those who did not [25]. The fourth new study prospectively investigated the use of DMPA compared with use of other contraceptive methods [31]. Most of the women studied received DMPA within the first 3 months postpartum. Those who received DMPA were more likely to be fully breastfeeding at 3 and 6 months postpartum and were more likely to continue breastfeeding through 12 and 18 months. Of women who received DMPA in the first 3 months, $35 \%$ were still breastfeeding at 12 months compared with $67 \%$ of those who received DMPA after 3 months.

The remaining studies were included previously. One found that no women using NET-EN, DMPA or nonhormonal methods supplemented breastfeeding in the 6-month study period [36]. Another found that women using DMPA breastfed for longer than a historical control group, although no difference was noted compared with IUD users [37]. Two other studies similarly found longer duration of breastfeeding among women using DMPA compared with nonhor- monal methods [38,39]. Another found that NET-EN and (presumably nonhormonal) IUD users had no difference in time to first supplementary feeding, but infants of IUD users weaned earlier [40]. Mothers who received either DMPA or a nonhormonal method at hospital discharge had no differences in breastfeeding exclusivity, supplementation or duration [41]. Finally, when DMPA initiated at hospital discharge was compared with nonhormonal method use, no differences were found in breastfeeding at 2 or 6 weeks, although fewer DMPA users were breastfeeding at 4 weeks [42].

3.1.2.2. POPs. Eight observational studies assessed the use of POPs in the first 6 weeks postpartum; all were included in the previous review and found either no differences between POP users and nonusers or improved breastfeeding outcomes with POP use. In a nonrandomized trial, POP users initiated breastfeeding earlier than placebo users [43]. Other studies found no difference in breastfeeding duration for POP users compared with historical controls [37] or compared with nonhormonal users [39,44], while two found longer breastfeeding duration among POP users compared with historical controls [45] or IUD users [46]. Finally, two studies found less supplementation among POP users than nonhormonal users $[47,48]$.

3.1.2.3. Implants. Five observational studies, all in the previous review, largely found no difference in outcomes when assessing the impact of implants in the first 6 weeks postpartum. Women using a norethindrone implant were more likely to supplement breastfeeding at 3 months than those using condoms, but no differences were noted at any other time through 6 months or in the mean duration of breastfeeding [49]. Two studies found no difference in supplementation comparing LNG implant with IUD users $[40,50]$; one of these also found no difference in breastfeeding duration [50]. Users of nomegestrol implants compared with IUD users similarly had no difference in time of weaning or breastfeeding rates through 12 months [51]. Finally, breastfeeding duration did not differ between users of an etonogestrel (ETG) implant compared with $\mathrm{Cu}$-IUD users over 3 years $[52,53]$.

3.1.2.4. Multiple POCs. One study, included previously, assessed users of the LNG implant or POPs (analyzed together) and found no differences in breastfeeding initiation or exclusivity, although POC users were less likely to be breastfeeding than nonhormonal users at one of three time points [42].

3.1.2.5. Nonorally available progestogens. Progesterone, unlike progestogens, is not absorbable orally; therefore, use during breastfeeding is believed to be safe for a neonate. As it is absorbed by the mother, it could impact breastfeeding. Two studies examined the use of progesterone pellets in the first 6 weeks postpartum; both were included in the previous 
review. Neither showed an impact on continuation of breastfeeding at 6 months [54] or at 6 and 12 months [55], compared with $\mathrm{Cu}$-IUD use.

\subsection{Initiation at $\geq 6$ weeks postpartum: Lactation performance}

One RCT and 13 observational studies (four newly identified $[24,26,28,30])$ evaluated the use of POCs initiated 6 weeks postpartum or more. None of these reported negative impacts on breastfeeding outcomes among POC users compared with nonusers, with the exception of one observational study that found that the average age of supplementation was younger among POP users compared with IUD users [30].

\subsubsection{RCTs}

One RCT, previously reviewed, found no difference between $\mathrm{Cu}-\mathrm{IUD}$ and LNG-IUD users in duration of breastfeeding or supplementation at 6-8 weeks postpartum [56].

\subsubsection{Observational studies}

3.2.2.1. Injectables. Three observational studies (one new) were identified. The new study did not find supplementation among infants of mothers receiving injectables nor among those who received no method [36]. Among the studies included in the previous review, one found no difference between DMPA and nonhormonal users in breastfeeding discontinuation or initiation of complementary foods [57]. Another found no difference in breastfeeding duration within study sites between DMPA and NET-EN users, compared with nonhormonal method users, although differences were seen between sites $[58,59]$.

3.2.2.2. POPs. Four studies (one new) assessed the impact of POPs on breastfeeding outcomes. In the new study, a nonrandomized trial [30], women used POPs, a $\mathrm{Cu}$-IUD plus placebo pill or one of several combined hormonal methods. The average age of supplementation was lower in the POP group compared with the IUD group (11.2 vs. 15 weeks), although statistical comparisons were not reported. Among the studies included in the prior review, one found no difference in complementary feeding or breastfeeding continuation up to 24 weeks when comparing POP users with nonhormonal users [57]. Two others found no difference in breastfeeding duration between POP and nonhormonal users [58-60].

3.2.2.3. Implants and hormonal IUDs. Six studies, two of which are new, assessed the impact of implant or hormonal IUD use on breastfeeding outcomes; none found differences between groups. One new study assessed the effect of both the ETG implant and the LNG-IUD compared with Cu-IUD and found no differences between groups in mean duration of breastfeeding at 6 months [24]. The other new study included LNG implant and Cu-IUD users and found no difference in the percentage of fully breastfeeding at month 6 or 12 and no difference in breastfeeding duration [28].
In one of the previously reviewed studies, women who received a norethindrone implant were similar to condom users in supplementation and breastfeeding continuation to 8 months [49]. In three studies of women who initiated the LNG implant, similar duration of breastfeeding was seen among both hormonal and nonhormonal users [58-62].

\subsubsection{Multiple progestogen-only methods. One new} study assessed the impact of multiple POCs without presenting outcomes separately by method. This prospective cohort found no difference in duration of breastfeeding between users of POCs and nonhormonal methods over 6 months [26].

3.2.2.5. Nonorally available progestogens. Three studies, none new, assessed the impact of nonorally available progestogens (progesterone pellets and nesterone or elcometrine implants) on breastfeeding outcomes. In two of these, breastfeeding duration was similar between women using progesterone pellets [54] or a nesterone implant [63], compared with $\mathrm{Cu}$-IUD. Elcometrine implant users had a higher rate of breastfeeding at 3 and 6 months and similar rates at 9-12 months compared with $\mathrm{Cu}-\mathrm{IUD}$ users [64].

\subsection{Initiation less than 6 weeks postpartum: Infant outcomes}

Thirty-seven studies (four RCTs, 32 observational studies and one cohort study with a nested RCT) were identified, including many of the studies previously described. Although some studies found differences in growth, health or development at some individual time points, most demonstrated no adverse impact of POCs.

\subsubsection{RCTs}

Three trials were identified. One of these studies is new and provides indirect evidence; the other two were included in the previous review. In the new study, women initiated either POPs or COCs at 2 weeks postpartum; no differences emerged in infant weight, length or head circumference through 8 weeks [21]. In a study of POPs or placebo, no differences were reported for infant weight gain at 14 days [33]. Similarly, infants of LNG-IUD users had similar weight, height and health through 12 months compared with $\mathrm{Cu}$-IUD users [34].

\subsubsection{Observational studies}

3.3.2.1. Injectables. Seven observational studies, three newly identified [27,29,32], assessed infant outcomes after initiation of POIs; all either found no detrimental effect or a protective effect of injectables on infant growth and health. A new cohort study of 250 women found no differences in infant growth or reports of illness up to 6 months when comparing users of DMPA initiated within 10 days postpartum with users of nonhormonal methods [27]. Another newly identified cohort study found no difference in infant weight gain up to 46 months between infants whose 
mothers had been exposed to DMPA at various time points and those who did not receive DMPA prior to 9 months postpartum [32]. No significant differences were found between groups in infant infections, although a subgroup that received DMPA within 2 days postpartum had a $75 \%$ higher incidence than the other groups (statistics not reported). Another cohort study included infants who were exposed to DMPA during breastfeeding (but not during their mother's pregnancy), during both pregnancy and breastfeeding or not at all [29]. Infants who were exposed only during breastfeeding were no more likely than the unexposed to have a height or weight over two standard deviations below the mean. Infants exposed to DMPA during breastfeeding (including those exposed during pregnancy) were more likely to have short stature; this difference was no longer significant after adjusting for socioeconomic factors and no effect on weight was seen. Follow-up period was unspecified.

The remaining four studies were included in the previous review. In one, infant weight gain was the same for NET-EN, DMPA and Cu-IUD users up to month 3, after which weight gain was greater in both the DMPA and NET-EN groups [36]. No physical, mental or radiological differences were seen through 18 months. Another study found no effect of maternal DMPA use on infant weight, development or health compared with nonhormonal method use through 3-6 years of follow-up [38]. One child death was reported in the nonhormonal group, and none was reported in the DMPA group. In another study, infants had no adverse effects with maternal NET-EN use through 30 months of age when compared with nonhormonal method use; specific outcomes were not provided [39]. The fourth study found no difference in growth or development among infants of NET-EN users compared with infants of $\mathrm{Cu}$-IUD users over 12 months [40].

3.3.2.2. POPS. Six observational studies or nonrandomized trials, none new in this review, assessed infant outcomes associated with POP use; most found no adverse effects. In one study, infants of women using POPs had greater weight increase than placebo users at day 14 [43]. Another study found no difference in urinary FSH, LH or testosterone among male infants of POP users compared with users of no method at 4 weeks [65]. Another study found no adverse effects of POPs up to an average of 4.5 years of age, compared with infants of women who used the lactational amenorrhea method (LAM) or the IUD (presumably nonhormonal) [39]. Two studies found no growth differences between infants of mothers using POPs compared with nonhormonal users $[47,48]$; one of these also found no difference in hospitalizations [47], while the other found more frequent minor illnesses and greater mortality ( 3 vs. 0 deaths) among children of mothers who used nonhormonal methods [48]. Finally, infants of desogestrel users had temporary breast enlargement (2 infants) and perceived increased sweating (1 infant), compared with no adverse effects among infants of Cu-IUD users [46].
Follow-up through 2.5 years revealed no clinically relevant effects of desogestrel on the growth or health of the infants.

3.3.2.3. Implants. Eight studies that assessed the impact of implants were included, none of which is new; generally no adverse effects were reported. In one study, infant weight was no different between norethindrone implant and barrier method users [49]. Another found no health or serum immunoglobulin differences between infants of LNG implant and nonhormonal users [66] and another found no differences in mean FSH, LH or testosterone [65]. One study found slower weight gain in infants of LNG implant users up to 3 months, compared with $\mathrm{Cu}$-IUD users. This difference disappeared at 4-6 months; however, length increased less among infants of LNG users compared with $\mathrm{Cu}$-IUD users [50]. No differences in morbidity were reported. In another study, infant lengths did not differ and weight was greater among infants of LNG implant users [67], and in a third, no differences were found between implant users and nonhormonal users in growth or development [40]. A study of the nomegestrol implant compared with $\mathrm{Cu}$-IUD found no difference in growth or health; greater infant mortality was seen in the implant group (six deaths from gastroenteritis, seizures and pneumonia, compared with one death from gastroenteritis in the $\mathrm{Cu}$-IUD group) but was not statistically significant [51]. Finally, a study of ETG implants compared with the $\mathrm{Cu}$-IUD found no differences in infant growth, adverse events, respiratory or skin disorders or developmental scores $[52,53]$.

3.3.2.4. Nonorally available progestogens. Two studies reported no difference in infant growth or health comparing progesterone pellet users with placebo or $\mathrm{Cu}$ IUD users $[54,55]$.

\subsection{Initiation at $\geq 6$ weeks: Infant outcomes}

Most of the studies described above also reported on infant outcomes. The majority found no significant differences between infants of POC users and nonhormonal method users, although differences in both directions were noted in some comparisons.

\subsection{1. $R C T s$}

Two RCTs (neither new) investigated the effect of POC initiation after 6 weeks postpartum. In both, no differences in infant growth or development were seen between users of the LNG-IUD compared with the Cu-IUD through 1 year [56] or between users of POPs or DMPA compared with nonhormonal method users through 24 weeks [57].

\subsubsection{Observational studies}

3.4.2.1. Injectables. Three observational studies (none new) assessed the impact of maternal use of POIs initiated at 6 weeks postpartum or later; none is new. One found increased weight gain among infants of DMPA and NET-EN 
users compared with nonhormonal users and also found no physical, mental or radiological differences over 18 months [36]. Another similarly found no difference in mean weight between DMPA users and nonhormonal users through 24 months [57]. Another study showed more weight gain among infants of DMPA and NET-EN users at some time points ( 3 and 12 months) and no difference at others (6 and 9 months). The majority of comparisons in developmental tests were similar, although some tests favored nonhormonal methods and others favored DMPA or NET-EN $[58,59]$.

3.4.2.2. POPs. Four studies, one new [30], assessed the impact of POPs. The new study found no difference in infant growth between those whose mothers used a POP compared with those whose mothers used an IUD plus placebo pill up to 32 weeks [30]. In the other three studies, one found smaller increase in arm circumference at two sites among infants of POP users compared with nonhormonal users but found no difference for other growth measures or for the majority of developmental test results [58,59]. The second found no differences in infant weight gain over 6 months when comparing infants of POC users with those of users of multiple other hormonal and nonhormonal methods [60]. The third found no difference in infant growth (length, weight, arm circumference) among infants of women using POPs compared with those using nonhormonal methods [57]

3.4.2.3. Implants/hormonal IUDs. Six studies, two new $[24,28]$, assessed infants whose mothers initiated progestogen-only implants or hormonal IUDs. In one new study, women initiated the ETG implant or LNG-IUD 6 weeks postpartum; no difference was found in infant weight or height through 6 months, although infants in the implant group had less increase in tibial length than infants in the $\mathrm{Cu}$-IUD group [24]. The other found no differences over 12 months in infant weight between users of the implant and users of the $\mathrm{Cu}$-IUD initiated at 8 weeks [28].

The remaining four studies were included previously. In one, infants of women who used a norethindrone implant had no differences in weight gain compared with those who used nonhormonal implants [49]. Likewise infants of LNG implant users had similar growth and development compared with nonhormonal users, although a few differences were noted in some of the multiple developmental tests $[58,59]$. In two studies of LNG implant use compared with nonhormonal methods, no differences were noted between groups in infant weight gain $[60,62]$, although in one of the studies, a higher incidence of respiratory infections and skin conditions was noted among infants whose mothers used an LNG implant [62]; more urologic and neurological conditions occurred among infants of $\mathrm{Cu}$-IUD users.

3.4.2.4. Multiple progestogen-only methods. One study (not new) included infants of mothers using various POCs and found that infant growth was generally the same between POC and nonhormonal users [68].
3.4.2.5. Nonorally available progestogens. Three studies reported on infant outcomes of mothers using nonorally available progestogens, none new. Infant growth was no different in users of nesterone pellets compared with nonhormonal methods [63]; neither infant growth nor health was different between users of progesterone pellets and users of nonhormonal methods [54]. Similarly, there was no difference in infant growth or development between infants of users of nesterone implants and Cu-IUD users [64].

\subsection{Key Question Two: Early versus delayed initiation}

In total, eight studies address the effect of initiation of POCs before 6 weeks postpartum compared with later initiation, of which five are new $[16,20,22,23,32]$. The majority found no effect on breastfeeding or infant outcomes, although one RCT found that more women continued breastfeeding at 6 months in the later initiation group [22] and another found more infections in infants of DMPA users [32].

\subsubsection{Breastfeeding outcomes: RCTs and observational studies}

Six of the studies assessed breastfeeding outcomes when POCs were initiated early or late postpartum (three RCTs, three observational). All three RCTs and one of the observational studies are new. One RCT compared women using ETG implants immediately postpartum versus DMPA initiated at 6 weeks [20]. No differences were seen between groups in the percentage of women exclusively breastfeeding at 6 or 12 weeks. Another RCT compared postplacental placement of LNG-IUD with delayed placement at 68 weeks and found no difference in breastfeeding initiation between groups or in breastfeeding continuation at 68 weeks; women in the delayed group were more likely to be breastfeeding at 6 months [22]. A final study compared women randomly assigned to the ETG implant either 13 days postpartum or at $4-8$ weeks postpartum and found no significant difference in breastfeeding outcomes [23].

One new observational study found that women who initiated DMPA after $72 \mathrm{~h}$ postpartum were more likely to be breastfeeding at 3 months than those who initiated before $72 \mathrm{~h}$ or who did not use DMPA [16]. In the other observational studies, norethindrone implant initiated early compared with delayed was not associated with differences in supplementary feeding or continuation of breastfeeding [49], and women who initiated progesterone pellets later were more likely to be supplementing breastfeeding than those who initiated early [54].

\subsubsection{Infant outcomes: Observational studies}

No RCTs and four observational studies (one new [32]) were identified for infant outcomes. The new study found that women who received DMPA within $48 \mathrm{~h}$ postpartum reported a higher incidence of infectious diseases in their infants than those who initiated DMPA later or not at all [32]. 
Among previously included studies, early versus delayed DMPA or NET-EN was not associated with any differences in growth, development or health [36], nor was early versus delayed norethindrone associated with growth differences [49]. Use of progesterone pellets was not associated with any differences in growth, development or health [54].

\section{Discussion}

Overall, evidence from 49 articles reporting on 47 studies on use of POCs during breastfeeding is of poor to fair methodological quality. Of the 14 studies that were newly included in this review, four were older studies [29-32] of poor quality and one was published in 1999 and of fair quality [28]. None of these older studies showed any negative effect of use of POCs on breastfeeding or infant outcomes. Of the nine studies that were published since the last review, four were RCTs. One of the four trials suggested that early, compared with delayed, postpartum initiation of the LNG-IUD was associated with shorter breastfeeding duration and less breastfeeding exclusivity at 6 months [22]. However, two other RCTs found no differences [20,23]. The fourth new trial provides indirect evidence demonstrating no difference in outcomes between POPs compared with COCs [21]. Among the newly identified observational studies, findings were generally consistent with the observational studies in the previous review, with no adverse effects noted on breastfeeding or infant outcomes.

Exogenous administration of POCs could theoretically inhibit breastfeeding [69]; however, the evidence in this review does not generally support a negative impact on breastfeeding outcomes. Studies examining the initiation of POCs among postpartum women overall demonstrated no adverse effects on measures of breastfeeding success, such as duration of breastfeeding or time to supplementation, although a few reported differences in both positive and negative directions at individual time points. The preponderance of the evidence points toward no deleterious impact of POCs on breastfeeding success, although further study is warranted to examine the impact of immediate postpartum placement of the LNG-IUD.

Theoretical concerns also have been raised regarding the impact of exposure to progestogens on neonates, particularly in the first 6 weeks of life [7]. Studies identified in this review showed no consistent adverse effects of exposure to progestogens through breast milk on infant health outcomes such as growth, development and health through the first few years of life. We identified no data to inform a conclusion on longer-term effects and any such effects remain unknown.

The PVR was not addressed in this review. A recent review concluded that PVR use among breastfeeding women did not affect breastfeeding performance or infant growth during the first year postpartum [18].

Our ability to draw firm conclusions is limited as most studies are observational, have lacked clear definitions of breastfeeding patterns and failed to control for potential confounders [70]. Many did not provide information key to determining their quality and did not perform tests of significance. Some were not informative to our cutoff point of 6 weeks as participants initiated both before 6 weeks and after. Initiation before 6 weeks ranged from immediately postpartum to nearly 42 days.

In 2014, the WHO Expert Working Group reviewed this evidence to evaluate medical eligibility criteria for the use of POCs among breastfeeding women. All of the abovementioned studies were reviewed with the exception of one, which was identified after the meeting and found no deleterious effects of POCs [27]. The findings of this systematic review were incorporated into the recent update of the MEC [71].

\section{Conclusion}

Consistent evidence by multiple measures of successful breastfeeding, largely from fair or poor quality observational studies, suggests that POCs, when used by lactating women, do not compromise a woman's ability to breastfeed. Evidence that POCs do not adversely affect infant growth, health or development during the first year postpartum is generally consistent across observational and randomized studies. Further research is necessary to determine any effects on child health or development beyond the first year. Evidence newly added to this review is largely consistent with the previous evidence.

Supplementary data to this article can be found online at http://dx.doi.org/10.1016/j.contraception.2015.09.010.

\section{Acknowledgements}

The authors would like to acknowledge the contributions of Mary Lyn Gaffield, Roger Chou and the Guidelines Development Group for the Medical Eligibility for Contraceptive Use. This review was supported by the WHO, the US Centers for Disease Control and Prevention and FHI 360.

\section{References}

[1] WHO Collaborative Study Team on the Role of Breastfeeding on the Prevention of Infant Mortality. Effect of breastfeeding on infant and child mortality due to infectious diseases in less developed countries: a pooled analysis. Lancet 2000;355:451-5.

[2] Kramer MS, Kakuma R. Optimal duration of exclusive breastfeeding. Cochrane Database Syst Rev 2012;8:Cd003517.

[3] Ip S, Chung M, Raman G, Chew P, Magula N, Devine D, Trikalinos T, et al. Breastfeeding and maternal and infant health outcomes in developed countries. Evid Rep Technol Assess 2007:1-86.

[4] World Health Organization. The Optimal Duration of Exclusive Breastfeeding. Geneva, Switzerland: WHO; 2001.

[5] Borda MR, Winfrey W, McKaig C. Return to sexual activity and modern family planning use in the extended postpartum period: an analysis of findings from seventeen countries. Afr J Reprod Health 2010;14:72-9. 
[6] Hatcher RA, Trussell J, Nelson AL. Contraceptive technology: Ardent Media; 2007

[7] Kennedy KI, Short RV, Tully MR. Premature introduction of progestin-only contraceptive methods during lactation. Contraception 1997:55:347-50.

[8] Rodriguez MI, Kaunitz AM. An evidence-based approach to postpartum use of depot medroxyprogesterone acetate in breastfeeding women. Contraception 2009;80:4-6.

[9] Diaz S. Contraceptive implants and lactation. Contraception 2002;65:39-46.

[10] Quadros PS, Pfau JL, Wagner CK. Distribution of progesterone receptor immunoreactivity in the fetal and neonatal rat forebrain. J Comp Neurol 2007;504:42-56.

[11] Wagner CK. The many faces of progesterone: a role in adult and developing male brain. Front Neuroendocrinol 2006;27:340-59.

[12] World Health Organization. Medical Eligibility Criteria for Contraceptive Use: A WHO Family Planning Cornerstone. 4th edit. Geneva, Switzerland: WHO; 2010.

[13] Kapp N, Curtis K, Nanda K. Progestogen-only contraceptive use among breastfeeding women: a systematic review. Contraception 2010;82:17-37.

[14] Liberati A, Altman DG, Tetzlaff J, Mulrow C, Gotzsche PC, Ioannides JP, et al. The PRISMA statement for reporting systematic reviews and meta-analyses of studies that evaluate healthcare interventions: explanation and elaboration. BMJ 2009;339:b2700.

[15] Croxatto HB, Díaz S, Peralta O, Juez G, Herreros C, Casado ME, et al. Fertility regulation in nursing women: IV. Long-term influence of a low-dose combined oral contraceptive initiated at day 30 postpartum upon lactation and infant growth. Contraception 1983;27:13-25.

[16] Matias SL, Nommsen-Rivers LA, Dewey KG. Determinants of exclusive breastfeeding in a cohort of primiparous periurban Peruvian mothers. J Hum Lact 2012;28:45-54.

[17] Nath A, Sitruk-Ware R. Progesterone vaginal ring for contraceptive use during lactation. Contraception 2010;82:428-34.

[18] Carr SL, Gaffield ME, Dragoman MV, Phillips SJ. Safety of the progesterone-releasing vaginal ring (PVR) among lactating women: a systematic review. Contraception 2016;94:253-61.

[19] Harris RP, Helfand M, Woolf SH, et al. Current methods of the US Preventive Services Task Force: a review of the process. Am J Prev Med 2001;20:21-35.

[20] Brito MB, Ferriani RA, Quintana SM, Yazlle ME, Silva de Sá MF, Vieira CS. Safety of the etonogestrel-releasing implant during the immediate postpartum period: a pilot study. Contraception 2009;80:519-26.

[21] Espey E, Ogburn T, Leeman L, Singh R, Ostrom K, Schrader R. Effect of progestin compared with combined oral contraceptive pills on lactation: a randomized controlled trial. Obstet Gynecol 2012;119:5-3.

[22] Chen BA, Reeves MF, Creinin MD, Schwarz EB. Postplacental or delayed levonorgestrel intrauterine device insertion and breast-feeding duration. Contraception 2011;84:499-504.

[23] Gurtcheff SE, Turok DK, Stoddard G, Murphy PA, Gibson M, Jones KP. Lactogenesis after early postpartum use of the contraceptive implant: a randomized controlled trial. Obstet Gynecol 2011;117:1114-21.

[24] Bahamondes L, Bahamondes MV, Modesto W, Tilley IB, Magalhães A, Pinto e Silva JL, et al. Effect of hormonal contraceptives during breastfeeding on infant's milk ingestion and growth. Fertil Steril 2013;100:445-50.

[25] Brownell EA, Fernandez ID, Fisher SG, Howard CR, Ternullo SR, Lawrence RA, et al. The effect of immediate postpartum depot medroxyprogesterone on early breastfeeding cessation. Contraception 2013;87:836-43.

[26] Costa ML, Cecatti JG, Krupa FG, Rehder PM, Sousa MH, Costa-Paiva L. Progestin-only contraception prevents bone loss in postpartum breastfeeding women. Contraception 2012;85:374-80.

[27] Singhal S, Sarda N, Gupta S, Goel S. Impact of injectable progestogen contraception in early puerperium on lactation and infant health. J Clin Diagn Res 2014;8:69-72.
[28] Díaz S, Reyes MV, Zepeda A, González GB, López JM, Campino C, et al. Norplant((R)) implants and progesterone vaginal rings do not affect maternal bone turnover and density during lactation and after weaning. Hum Reprod 1999;14:2499-505.

[29] Pardthaisong T, Yenchit C, Gray R. The long-term growth and development of children exposed to Depo-Provera during pregnancy or lactation. Contraception 1992;45:313-24.

[30] Kamal I, Hefnawi F, Ghoneim M, Talaat M, Younis N, Taqui A, et al. Clinical, biochemical, and experimental studies on lactation. II. Clinical effects of gestagens on lactation. Am J Obstet Gynecol 1969;105:324-34.

[31] Zañartu J, Aguilera E, Munoz G, Peliowsky H. Effect of a long-acting contraceptive progestogen on lactation. Obstet Gynecol 1976;47: $174-6$.

[32] Dahlberg K. Some effects of depo-medroxyprogesterone acetate (DMPA): observations in the nursing infant and in the long-term user. Int J Gynaecol Obstet 1982;20:43-8.

[33] Giner Velazquez J, Cortes Gallegos V, Sotelo Lopez A, Bondani G. Effect of daily oral administration of $0.350 \mathrm{mg}$ of norethindrone on lactation and on the composition of milk. Ginecol Obstet Mex 1976;40:31-9.

[34] Heikkila M, Luukkainen T. Duration of breast-feeding and development of children after insertion of a levonorgestrel-releasing intrauterine contraceptive device. Contraception 1982;25:279-92.

[35] Lawrie TA, Hofmeyr GJ, De Jager M, Berk M, Paiker J, Viljoen E. A double-blind randomised placebo controlled trial of postnatal norethisterone enanthate: the effect on postnatal depression and serum hormones. Br J Obstet Gynaecol 1998;105:1082-90.

[36] Karim M, Ammar R, el-Mahgoub S, el-Ganzoury B, Fikri F, Abdou I. Injected progestogen and lactation. Br Med J 1971;1:200-3.

[37] Guiloff E, Ibarra-Polo A, Zañartu J, Toscanini C, Mischler TW, Gómez-Rogers C. Effect of contraception on lactation. Am J Obstet Gynecol 1974;118:42-5.

[38] Jimenez J, Ochoa M, Soler MP, Portales P. Long-term follow-up of children breast-fed by mothers receiving depot-medroxyprogesterone acetate. Contraception 1984;30:523-33.

[39] Zacharias S, Aguilera E, Assenzo JR, Zañartu J. Effects of hormonal and nonhormonal contraceptives on lactation and incidence of pregnancy. Contraception 1986;33:203-13

[40] Shaaban MM. Contraception with progestogens and progesterone during lactation. J Steroid Biochem Mol Biol 1991;40:705-10.

[41] Hannon PR, Duggan AK, Serwint JR, Vogelhut JW, Witter F, DeAngelis C. The influence of medroxyprogesterone on the duration of breast-feeding in mothers in an urban community. Arch Pediatr Adolesc Med 1997;151:490-6.

[42] Halderman LD, Nelson AL. Impact of early postpartum administration of progestin-only hormonal contraceptives compared with nonhormonal contraceptives on short-term breast-feeding patterns. Am J Obstet Gynecol 2002;186:1250-6.

[43] Kamal I, Hefnawi F, Ghoneim M, Abdallah M, Abdel Razek S. Clinical, biochemical, and experimental studies on lactation. V. Clinical effects of steroids on the initiation of lactation. Am J Obstet Gynecol 1970;108:655-8.

[44] West CP. The acceptability of a progestagen-only contraceptive during breast-feeding. Contraception 1983;27:563-9.

[45] Zañartu J, Aguilera E, Munoz-Pinto G. Maintenance of lactation by means of continuous low-dose progestogen given post-partum as a contraceptive. Contraception 1976;13:313-8

[46] Bjarnadottir RI, Gottfredsdottir H, Sigurdardottir K, Geirsson RT, Dieben TO. Comparative study of the effects of a progestogen-only pill containing desogestrel and an intrauterine contraceptive device in lactating women. BJOG 2001;108:1174-80.

[47] McCann MF, Moggia AV, Higgins JE, Potts M, Becker C. The effects of a progestin-only oral contraceptive (levonorgestrel $0.03 \mathrm{mg}$ ) on breast-feeding. Contraception 1989;40:635-48.

[48] Moggia AV, Harris GS, Dunson TR, Diaz R, Moggia MS, Ferrer MA, et al. A comparative study of a progestin-only oral contraceptive versus 
non-hormonal methods in lactating women in Buenos Aires, Argentina. Contraception 1991;44:31-43.

[49] Seth U, Yadava HS, Agarwal N, Laumas KR, Hingorani V. Effect of a subdermal silastic implant containing norethindrone acetate on human lactation. Contraception 1977;16:383-98.

[50] Shaaban MM, Salem HT, Abdullah KA. Influence of levonorgestrel contraceptive implants, NORPLANT, initiated early postpartum upon lactation and infant growth. Contraception 1985;32:623-35.

[51] Abdel-Aleem H, Abol-Oyoun el SM, Shaaban MM, el-Saeed M, Shoukry M, Makhlouf A, et al. The use of nomegestrol acetate subdermal contraceptive implant, uniplant, during lactation. Contraception 1996;54:281-6.

[52] Reinprayoon D, Taneepanischskul S, Bunyavejchevin S, Thaithumyanon $\mathrm{P}$, Punnahitananda $\mathrm{S}$, Tosukhowong $\mathrm{P}$, et al. Effects of the etonogestrel-releasing contraceptive implant (Implanon on parameters of breastfeeding compared to those of an intrauterine device). Contraception 2000;62:239-46.

[53] Taneepanichskul S, Reinprayoon D, Thaithumyanon P, Praisuwanna $\mathrm{P}$, Tosukhowong P, Dieben T. Effects of the etonogestrel-releasing implant Implanon and a nonmedicated intrauterine device on the growth of breast-fed infants. Contraception 2006;73:368-71.

[54] Díaz S, Peralta O, Juez G, et al. Fertility regulation in nursing women. VI. Contraceptive effectiveness of a subdermal progesterone implant. Contraception 1984;30:311-25.

[55] Croxatto HB, Díaz S, Peralta O, Juez G, Casado ME, Salvatierra AM, et al. Fertility regulation in nursing women. II. Comparative performance of progesterone implants versus placebo and copper T. Am J Obstet Gynecol 1982;144:201-8.

[56] Shaamash AH, Sayed GH, Hussien MM, Shaaban MM. A comparative study of the levonorgestrel-releasing intrauterine system Mirena versus the Copper T380A intrauterine device during lactation: breast-feeding performance, infant growth and infant development. Contraception 2005; $72: 346-51$.

[57] Tankeyoon M, Dusitsin N, Chalapati S, Koetsawang S, Saibiang S, Sas M, et al. Effects of hormonal contraceptives on milk volume and infant growth. WHO Special Programme of Research, Development and Research Training in Human Reproduction Task force on oral contraceptives. Contraception 1984;30:505-22.

[58] Progestogen-only contraceptives during lactation: I. Infant growth. World Health Organization Task force for Epidemiological Research on Reproductive Health; Special Programme of Research, Development and Research Training in Human Reproduction. Contraception 1994;50:35-53.

[59] Progestogen-only contraceptives during lactation: II. Infant development. World Health Organization, Task Force for Epidemiological
Research on Reproductive Health; Special Programme of Research, Development, and Research Training in Human Reproduction. Contraception 1994;50:55-68.

[60] Díaz S, Zepeda A, Maturana X, Reyes MV, Miranda P, Casado ME, et al. Fertility regulation in nursing women. IX. Contraceptive performance, duration of lactation, infant growth, and bleeding patterns during use of progesterone vaginal rings, progestin-only pills, Norplant implants, and Copper T 380-A intrauterine devices. Contraception 1997;56:223-32.

[61] Díaz S, Herreros C, Juez G, et al. Fertility regulation in nursing women: VII. Influence of Norplant levonorgestrel implants upon lactation and infant growth. Contraception 1985;32:53-74.

[62] Schiappacasse V, Díaz S, Zepeda A, Alvarado R, Herreros C. Health and growth of infants breastfed by Norplant contraceptive implants users: a six-year follow-up study. Contraception 2002;66:57-65.

[63] Massai MR, Díaz S, Quinteros E, Reyes MV, Herreros C, Zepeda A, et al. Contraceptive efficacy and clinical performance of Nestorone implants in postpartum women. Contraception 2001;64:369-76.

[64] Coutinho EM, Athayde C, Dantas C, Hirsch C, Barbosa I. Use of a single implant of elcometrine (ST-1435), a nonorally active progestin, as a long acting contraceptive for postpartum nursing women. Contraception 1999;59:115-22.

[65] Shikary ZK, Betrabet SS, Toddywala WS, Patel DM, Datey S, Saxena BN. Pharmacodynamic effects of levonorgestrel (LNG) administered either orally or subdermally to early postpartum lactating mothers on the urinary levels of follicle stimulating hormone (FSH), luteinizing hormone ( $\mathrm{LH})$ and testosterone $(\mathrm{T})$ in their breast-fed male infants. Contraception 1986;34:403-12.

[66] Abdulla KA, Elwan SI, Salem HS, Shaaban MM. Effect of early postpartum use of the contraceptive implants, Norplant, on the serum levels of immunoglobulins of the mothers and their breastfed infants. Contraception 1985;32:261-6.

[67] Affandi B, Karmadibrata S, Prihartono J, Lubis F, Samil RS. Effect of Norplant on mothers and infants in the postpartum period. Adv Contracept 1986;2:371-80.

[68] Baheiraei A, Ardsetani N, Ghazizadeh S. Effects of progestogen-only contraceptives on breast-feeding and infant growth. Int J Gynaecol Obstet 2001;74:203-5.

[69] Brownell EA, Fernandez ID, Howard CR, Fisher SG, Ternullo SR, Buckley RJ, et al. A systematic review of early postpartum medroxyprogesterone receipt and early breastfeeding cessation: evaluating the methodological rigor of the evidence. Breastfeed Med 2012;7:10-8.

[70] Thulier D, Mercer J. Variables associated with breastfeeding duration. J Obstet Gynecol Neonatal Nurs 2009;38:259-68.

[71] World Health Organization. Medical Eligibility Criteria for Contraceptive Use. Geneva, Switzerland: WHO; 2015. 